\title{
Constructive Existence of $(1,1)$-Solutions to Two-Point Value Problems for Fuzzy Linear Multiterm Fractional Differential Equations
}

\author{
HuiChol Choi, ${ }^{1}$ SungHyok Kwon, ${ }^{1}$ Kinam Sin ${ }^{D},{ }^{1}$ Sunae Pak, ${ }^{1}$ and Sungryol So ${ }^{2}$ \\ ${ }^{1}$ Faculty of Mathematics, Kim Il Sung University, Pyongyang, Democratic People's Republic of Korea \\ ${ }^{2}$ Information Technology Institute, University of Sciences, Pyongyang, Democratic People's Republic of Korea \\ Correspondence should be addressed to Kinam Sin; 15bf12025@hit.edu.cn
}

Received 3 February 2019; Revised 10 May 2019; Accepted 22 May 2019; Published 17 June 2019

Academic Editor: Feyzi Başar

Copyright (C) 2019 HuiChol Choi et al. This is an open access article distributed under the Creative Commons Attribution License, which permits unrestricted use, distribution, and reproduction in any medium, provided the original work is properly cited.

In this paper, we consider the following two-point boundary value problems of fuzzy linear fractional differential equations: $\left({ }^{c} D_{1,1}^{\alpha} y\right)(t) \oplus b(t) \otimes\left({ }^{c} D_{1,1}^{\beta} y\right)(t) \oplus c(t) \otimes y(t)=f(t), t \in(0,1), y(0)=y_{0}$ and $y(1)=y_{1}$, where $b, c \in C(I), b(t), c(t) \geq 0$, $y, f \in C\left(I, \mathbf{R}_{\mathrm{F}}\right), I=[0,1], y_{0}, y_{1} \in \mathbf{R}_{\mathrm{F}}$ and $1<\beta<\alpha \leq 2$. Our existence result is based on Banach fixed point theorem and the approximate solution of our problem is obtained by applying the Haar wavelet operational matrix.

\section{Introduction}

A lot of researchers have studied fuzzy differential equations especially fuzzy boundary value problems (FBVPs) because they are effective tools for modeling processes. FBVPs arise in many applications such as modeling of fuzzy optimal control problem [1] and HIV infection [2]. Many theoretical researches have been carried out on fractional differential equations over the last years [3-7].

Currently, the approximative methods for solving fuzzy fractional differential equation include the operational matrix method based on orthogonal functions [8-10], linearization formula [11], Homotopy Analysis Method [12-14], and Variation of constant formula [15].

O'Regan [16] and Lakshmikantham [17] proved that twopoint boundary value problems of fuzzy differential equations are equivalent to fuzzy integral equations.

Lakshmikantham et al. [18] considered Riemann-Liouville differentiability concept based on the Hukuhara differentiability to solve fuzzy fractional differential equations. Prakash [19] considered initial value problems for differential equations of fractional order with uncertainty. Mazandarania [20] investigated the solution to fuzzy fractional initial value problem (FFIVP) under Caputo-type fuzzy fractional derivatives by a modified fractional Euler method. As we can see, fuzzy initial value problems were studied by many researchers, but few fuzzy boundary value problems were considered in special cases. Nieto [21] considered second order fuzzy differential by the sense of $(1,1),(1,2),(2,1)$, $(2,2)$-derivatives. Also Nieto [22] investigated the existence and uniqueness of solutions for a first-order linear fuzzy differential equation with impulsive boundary value condition. Ngo et al. [23] proved the existence and uniqueness results of the solution to initial value problem of Caputo-Katugampola (CK) fractional differential equations in fuzzy setting and [24] present that a fractional fuzzy differential equation and a fractional fuzzy integral equation are not equivalent in general. Wang [25] considered the existence and uniqueness of solution for a class of FFDEs:

$$
\begin{aligned}
C_{g H D_{*}^{q}} u(t) & =f(t, u(t)), \quad 0<t<T, \\
u(0) & =\lambda u(T), \\
u_{g H}^{\prime}(0) & =u_{0} \in E_{C}^{1},
\end{aligned}
$$

where ${ }^{C} g H D_{*}^{q}$ is the fuzzy $g H$-fractional Caputo derivative, $u_{g H}^{\prime}$ is generalized Hukuhara derivative of $u, E_{C}^{1}$ is the space of fuzzy number, $f:[0, T] \times E_{c}^{1} \longrightarrow E_{c}^{1} \backslash R$ is a continuous 
fuzzy valued function, $q \in(1,2]$ is a real number, and $\lambda \epsilon$ $(0,1) \cup(1,+\infty)$.

Gasilov [26] presented a new approach to a nonhomogeneous fuzzy boundary value problem.

But researchers who studied fuzzy differential equations by using $r$-cut did not consider if the solutions of $r$-cut equations constitute intervals. So they had to recheck whether the solutions of $r$-cut equations constitute intervals or not after solving a problem. For instance, in [27] they only consider the existence of solutions of $r$-cut equation. And the existence of fuzzy solutions was considered in specific example. In that specific example, they noted that the fuzzy solutions do not exist even if the solutions of $r$-cut equation exist.

These facts lead to the following: the existence of solutions of fuzzy problem is not equivalent to the existence of solutions of corresponding $r$-cut equation. So, it is necessary to obtain a new $r$-cut problem that guarantees the existence of fuzzy solutions.

Motivated by the results mentioned above, we consider the following fuzzy boundary value problem:

$$
\begin{aligned}
& \left({ }^{c} D_{1,1}^{\alpha} y\right)(t) \oplus b(t) \otimes\left({ }^{c} D_{1,1}^{\beta} y\right)(t) \oplus c(t) \otimes y(t) \\
& \quad=f(t), \quad t \in(0,1), \\
& y(0)=y_{0}, \\
& y(1)=y_{1},
\end{aligned}
$$

where $b, c \in C(I), b(t), c(t) \geq 0, y, f \in C\left(I, \mathbf{R}_{\mathrm{F}}\right), I=[0,1]$, $y_{0}, y_{1} \in \mathbf{R}_{\mathrm{F}}, 1<\beta<\alpha \leq 2,{ }^{c} D_{1,1}^{\alpha}$ is fuzzy fractional Caputo derivative, and $\mathbf{R}_{\mathrm{F}}$ is the space of fuzzy number.

We obtain existence result by using Banach fixed point theorem and obtain its approximate solution by applying the Haar wavelet operational matrix. Also we present a new $r$-cut problem which involves inequalities to obtain the conditions of existence of fuzzy solutions and prove that these inequalities guarantee that the solutions of $r$-cut equations constitute fuzzy solutions. Our paper is organized as follows: In Section 2, we recall some definitions and basic results and prove some lemmas that will be useful to our main results. Section 3 investigated the constructive existence of solutions to our problem. In Section 4, a method to find out the solutions is given. Section 5 presented two examples to illustrate our results. In Section 6, we summarize our main results.

\section{Preliminaries and Basic Results}

Definition 1 (see [28]). We denote the set of all fuzzy numbers on $\mathbf{R}$ by $\mathbf{R}_{\mathrm{F}}$. A fuzzy number is a mapping $\mu: \mathbf{R} \longrightarrow[0,1]$ with the following properties:

(i) $\mu$ is normal, i.e., $\exists x_{0} \in \mathbf{R} ; \mu\left(x_{0}\right)=1$

(ii) $\mu$ is a convex fuzzy subset, i.e.,

$$
\begin{aligned}
& \mu(\lambda x+(1-\lambda) y) \geq \min \{\mu(x), \mu(y)\} \\
& \forall x, y \in \mathbf{R}, \forall \lambda \in[0,1]
\end{aligned}
$$

(iii) $\mu$ is upper semicontinuous on $\mathbf{R}$

(iv) The set $\overline{\operatorname{supp}(\mu)}$ is compact in $\mathbf{R}($ where $\operatorname{supp}(\mu):=$ $\{x \in \mathbf{R} \mid \mu(x)>0\})$

Then $\mathbf{R}_{\mathrm{F}}$ is called the space of fuzzy numbers.

Definition 2 (see [27]). Let $u, v \in \mathbf{R}_{\mathrm{F}},[u]^{r}:=\left[u_{-}^{(r)}, u_{+}^{(r)}\right]$, $[v]^{r}:=\left[v_{-}^{(r)}, v_{+}^{(r)}\right]$. The distance structure $D: \mathbf{R}_{\mathrm{F}} \times \mathbf{R}_{\mathrm{F}} \longrightarrow \mathbf{R}_{+}$ is defined as follows:

$$
D(u, v):=\sup _{r \in[0,1]} \max \left\{\left|u_{-}^{(r)}-v_{-}^{(r)}\right|,\left|u_{+}^{(r)}-v_{+}^{(r)}\right|\right\} .
$$

Let $x, y \in \mathbf{R}_{\mathrm{F}}$. If there exists $z \in \mathbf{R}_{\mathrm{F}}$ such that $x=y \oplus z$, then $z$ is called the H-difference (Hukuhara difference) of $x, y$ and it is denoted as $x \Theta_{H} y$.

Definition 3 (see [29]). Let $f: I \longrightarrow \mathbf{R}_{\mathrm{F}}$ and fix $x_{0} \in(a, b)$.

(i) We say that $f$ is (1)-differentiable at $x_{0}$, if there exists an element $f_{G}^{\prime}\left(x_{0}\right) \in \mathbf{R}_{\mathrm{F}}$ such that, for all $h>0$ sufficiently near to $0, f\left(x_{0}+h\right) \Theta_{H} f\left(x_{0}\right), f\left(x_{0}\right) \Theta_{H} f\left(x_{0}-h\right)$ and the limits

$$
\begin{aligned}
\lim _{h \rightarrow 0^{+}} & \frac{f\left(x_{0}+h\right) \Theta_{H} f\left(x_{0}\right)}{h} \\
& =\lim _{h \rightarrow 0^{+}} \frac{f\left(x_{0}\right) \Theta_{H} f\left(x_{0}-h\right)}{h}=f_{G}^{\prime}\left(x_{0}\right)
\end{aligned}
$$

exist.

(ii) We say that $f$ is (2)-differentiable at $x_{0}$, if there exists an element $f_{G}^{\prime}\left(x_{0}\right) \in \mathbf{R}_{\mathrm{F}}$ such that, for all $h>0$ sufficiently near to $0, f\left(x_{0}\right) \Theta_{H} f\left(x_{0}+h\right), f\left(x_{0}-h\right) \Theta_{H} f\left(x_{0}\right)$ and the limits

$$
\begin{aligned}
\lim _{h \rightarrow 0^{+}} \frac{f\left(x_{0}\right) \Theta_{H} f\left(x_{0}+h\right)}{-h} \\
\quad=\lim _{h \rightarrow 0^{+}} \frac{f\left(x_{0}-h\right) \Theta_{H} f\left(x_{0}\right)}{-h}=f_{G}^{\prime}\left(x_{0}\right)
\end{aligned}
$$

exist.

If $f$ is $(n)$-differentiable at $x_{0}$, we denote its first derivatives by $D_{n}^{(1)} f\left(x_{0}\right)$ for $n=1,2$.

Lemma 4 (see [21]). Let $f:(a, b) \longrightarrow \mathbf{R}_{\mathrm{F}}$ be fuzzy valued function, where $[f(t)]^{r}:=\left[f_{1}(t, r), f_{2}(t, r)\right]$ for each $r \in[0,1]$.

(i) If $f$ is (1)-differentiable, then $f_{1}(t, r)$ and $f_{2}(t, r)$ are differentiable functions and $\left[D_{1}^{(1)} f(t)\right]^{r}=\left[f_{1}^{\prime}(t, r)\right.$, $\left.f_{2}^{\prime}(t, r)\right]$.

(ii) If $f$ is (2)-differentiable, then $f_{1}(t, r)$ and $f_{2}(t, r)$ are differentiable functions and $\left[D_{2}^{(1)} f(t)\right]^{r}=\left[f_{2}^{\prime}(t, r)\right.$, $\left.f_{1}^{\prime}(t, r)\right]$.

Definition 5 (see [21]). Let $f:(a, b) \longrightarrow \mathbf{R}_{\mathrm{F}}$ and $n, m=1,2$. We say that $f$ is $(n, m)$ - differentiable at $x_{0} \in(a, b)$, if $D_{n}^{(1)} f\left(x_{0}\right)$ exists, and is $(m)-$ differentiable at $x_{0}$. The second derivative of $f$ is denoted by $D_{n, m}^{(2)} f\left(x_{0}\right)$ for $n, m=1,2$.

Lemma 6 (see [30]). Let $f:(a, b) \longrightarrow \mathbf{R}_{\mathrm{F}}$ be fuzzy valued function and denote its $r$ - level sets by $\quad[f(t)]^{r}:=\left[f_{1}(t, r)\right.$, $\left.f_{2}(t, r)\right]$ for each $r \in[0,1]$. 
(i) If $D_{1}^{(1)} f(t)$ is (1)-differentiable, then $f_{1}^{\prime}(t, r), f_{2}^{\prime}(t, r)$ are differentiable and

$$
\left[D_{1,1}^{(2)} f(t)\right]^{r}=\left[f_{1}^{\prime \prime}(t, r), f_{2}^{\prime \prime}(t, r)\right] .
$$

(ii) If $D_{1}^{(1)} f(t)$ is (2)-differentiable, then $f_{1}^{\prime}(t, r), f_{2}^{\prime}(t, r)$ are differentiable and

$$
\left[D_{1,2}^{(2)} f(t)\right]^{r}=\left[f_{2}^{\prime \prime}(t, r), f_{1}^{\prime \prime}(t, r)\right] .
$$

Definition 7. The Riemann-Liouville fractional integral operator of order $\alpha>0$ of a real function $\varphi: I \longrightarrow \mathbf{R}$ is defined as

$$
I_{a+}^{\alpha} \varphi(t)=\frac{1}{\Gamma(\alpha)} \int_{a}^{t}(t-s)^{\alpha-1} \varphi(s) d s,
$$

where $\Gamma(\cdot)$ is the Euler gamma function.

Definition 8. Let $\varphi: I \longrightarrow \mathbf{R}$, the Caputo fractional derivative of order $\alpha>0, m-1<\alpha<m, m \in \mathbf{N}=\{1,2, \cdots\}$, be defined as

$$
\left({ }^{c} D_{a+}^{\alpha} \varphi\right)(t)=\frac{1}{\Gamma(m-\alpha)} \int_{a}^{t}(t-s)^{m-\alpha-1} \varphi^{(m)}(s) d s .
$$

Definition 9. The fuzzy fractional Caputo differentiability of fuzzy valued function is defined as follows:

$$
\left({ }^{c} D_{1,1}^{\alpha} f\right)(t)=\frac{1}{\Gamma(2-\alpha)} \int_{a}^{t}(t-s)^{1-\alpha}\left(D_{1,1}^{(2)} f\right)(s) d s,
$$

where $1<\alpha \leq 2, t>0, f: I \longrightarrow \mathbf{R}_{\mathrm{F}}$. Then we say that $f$ is ${ }^{c}[(1,1)-\alpha]$-differentiable.

Lemma 10 (see $[23,24,31])$. Let $f: I \longrightarrow \mathbf{R}_{\mathrm{F}}$, $\alpha \in(1,2), \quad[f(t)]^{r}:=\left[f_{1}(t, r), f_{2}(t, r)\right]$. If $f$ is ${ }^{c}[(1,1)-\alpha]-$ differentiable, it holds that

$$
\left[\left({ }^{c} D_{1,1}^{\alpha} f\right)(t)\right]^{r}=\left[{ }^{c} D_{0+}^{\alpha} f_{1}(t, r),{ }^{c} D_{0+}^{\alpha} f_{2}(t, r)\right] .
$$

Lemma 11 (see [27]). Let $\left\{U_{r} \mid r \in(0,1]\right\}$ be a family of real intervals such that the following three conditions are satisfied:

(i) $U_{r}$ is a nonempty compact interval for all $r \in[0,1]$

(ii) if $0<r_{1}<r_{2} \leq 1$ then $U_{r_{2}} \subseteq U_{r_{1}}$

(iii) given any nondecreasing sequence $r_{n} \in(0,1]$ with $\lim _{n \rightarrow \infty} r_{n}=r$ it is $U_{r}=\bigcap_{n=1}^{\infty} U_{r_{n}}$

Then there exists a unique fuzzy number $u \in \mathbf{R}_{\mathrm{F}}$ such that $[u]^{r}=U_{r}$ for all $r \in I$ and $[u]^{0}=\operatorname{cl}\left(\bigcup_{r \in(0,1]} U_{r}\right)$.

Let us consider the following fractional integral equation:

$$
U(t)+b(t) I_{0+}^{\lambda_{1}} U(t)+c(t) I_{0+}^{\lambda_{2}} U(t)=V(t), \quad t \in I,
$$

where $V, b, c \in C(I), \lambda_{1}, \lambda_{2}>0$.

Lemma 12. There exists a unique solution of (14) in C(I) and a positive real number $k_{*}$ for the solution to satisfy the following inequality:

$$
\|U\|_{C(I)} \leq \frac{e^{k_{*}}}{1-q}\|V\|_{C(I)},
$$

where $k_{*}$ is any positive number that satisfies $q:=\left(\|b\|_{C(I)} /\right.$ $\left.k_{*}^{\lambda_{1}}+\|c\|_{C(I)} / k_{*}^{\lambda_{2}}\right)<1$.

Proof. Let $F(U)(t):=V(t)-b(t) I_{0+}^{\lambda_{1}} U(t)-c(t) I_{0+}^{\lambda_{2}} U(t)$.

Then equation (14) is as follows:

$$
U(t)=F(U)(t) .
$$

Now, we use the following $k$ - norm equivalent to the norm $\|x\|_{C(I)}:=\max \{|x(t)|, t \in I\}$.

That is

$$
\|x\|_{k}:=\max \left\{e^{-k t}|x(t)|\right\}, \quad x \in C(I) .
$$

For all $X, Y \in C(I)$, we have the following inequalities:

$$
\begin{aligned}
& |F(X)(t)-F(Y)(t)| \\
& \leq\|b\|_{C(I)}\left|I_{0+}^{\lambda_{1}} X(t)-I_{0+}^{\lambda_{1}} Y(t)\right| \\
& \quad+\|c\|_{C(I)}\left|I_{0+}^{\lambda_{2}} X(t)-I_{0+}^{\lambda_{2}} Y(t)\right| \\
& \leq\left(\frac{\|b\|_{C(I)}}{k^{\lambda_{1}}}+\frac{\|c\|_{C(I)}}{k^{\lambda_{2}}}\right) e^{k t}\|X-Y\|_{k} .
\end{aligned}
$$

Therefore, we get

$$
\begin{aligned}
& e^{-k t}|F(X)(t)-F(Y)(t)| \\
& \quad \leq\left(\frac{\|b\|_{C(I)}}{k^{\lambda_{1}}}+\frac{\|c\|_{C(I)}}{k^{\lambda_{2}}}\right)\|X-Y\|_{k} .
\end{aligned}
$$

Thus, we obtain

$$
\begin{aligned}
& \|F(X)(t)-F(Y)(t)\|_{k} \\
& \quad \leq\left(\frac{\|b\|_{C(I)}}{k^{\lambda_{1}}}+\frac{\|c\|_{C(I)}}{k^{\lambda_{2}}}\right)\|X-Y\|_{k} .
\end{aligned}
$$

Since the number $k_{*}$ satisfies

$$
q:=\frac{\|b\|_{C(I)}}{k^{\lambda_{1}}}+\frac{\|c\|_{C(I)}}{k^{\lambda_{2}}}<1,
$$

we obtain the following inequality:

$$
\mid F(X)-F(Y)\left\|_{k_{*}} \leq q\right\| X-Y \|_{k_{*}} .
$$

Since $C(I)$ is complete, we have

$$
\exists \bar{Y} \in C(I) ; \quad \bar{Y}=F(\bar{Y}) .
$$

That is, (14) has unique solution $U(t) \in C(I)$.

Then the following equation holds:

$$
U(t)+b(t) I_{0+}^{\lambda_{1}} U(t)+c(t) I_{0+}^{\lambda_{2}} U(t)=V(t), \quad t \in I,
$$


and we obtain the following inequality:

$$
\begin{aligned}
|U(t)| & \leq\|b\| I_{0+}^{\lambda_{1}}|U(t)|+\|c\| I_{0+}^{\lambda_{2}}|U(t)|+|V(t)| \\
& \leq\|b\| \frac{e^{k t}}{k^{\lambda_{1}}}\|U\|_{k}+\|c\| \frac{e^{k t}}{k^{\lambda_{2}}}\|U\|_{k}+|V(t)|,
\end{aligned}
$$

so we have

$$
\begin{aligned}
e^{-k t}|U(t)| \leq & \|b\| \frac{1}{k^{\lambda_{1}}}\|U\|_{k}+\|c\| \frac{1}{k^{\lambda_{2}}}\|U\|_{k} \\
& +e^{-k t}|V(t)| .
\end{aligned}
$$

Therefore, we get

$$
\begin{aligned}
\|U\|_{k} & \leq\|b\| \frac{1}{k^{\lambda_{1}}}\|U\|_{k}+\|c\| \frac{1}{k^{\lambda_{2}}}\|U\|_{k}+\|V\|_{k} \\
& =\left(\|b\| \frac{1}{k^{\lambda_{1}}}+\|c\| \frac{1}{k^{\lambda_{2}}}\right)\|U\|_{k}+\|V\|_{k} .
\end{aligned}
$$

So if there is a positive number $k_{*}$ satisfying

$$
q:=\frac{\|b\|_{C(I)}}{k_{*}^{\lambda_{1}}}+\frac{\|c\|_{C(I)}}{k_{*}^{\lambda_{2}}}<1,
$$

then we get

$$
\|U\|_{k_{*}} \leq \frac{1}{1-q}\|V\|_{k_{*}}
$$

and so we obtain

$$
e^{-k_{*}}\|U\|_{C(I)} \leq\|U\|_{k_{*}} \leq \frac{1}{1-q}\|V\|_{k_{*}} \leq \frac{1}{1-q}\|V\|_{C(I)} .
$$

Thus we have

$$
\|U\|_{C(I)} \leq \frac{e^{k_{*}}}{1-q}\|V\|_{C(I)}
$$

Consider the integral equation

$$
U(t)+b I_{0+}^{\alpha-\beta} U(t)+c I_{0+}^{\alpha} U(t)=h(t), \quad t \in[0, T],
$$

where $b>0, c \geq 0,1<\beta<\alpha \leq 2,0<\alpha-\beta<1$.

We define an operator $A$ by $A(U)(t):=b I_{0+}^{\alpha-\beta} U(t)+$ $c I_{0+}^{\alpha} U(t)$.

Lemma 13. If $(I-A) h(t) \geq 0$, then the integral equation (32) has one nonnegative solution, where $I$ is the identity operator.

Proof. By Lemma 12, (32) has a unique solution. If there is a positive number $k_{*}$ satisfying

$$
q:=\frac{b}{k_{*}^{\alpha-\beta}}+\frac{c}{k_{*}^{\alpha}}<1,
$$

then $\|A\|_{k_{*}}<1$.
Therefore we can know that

$$
(I+A)^{-1}=I-A+A^{2}-\cdots+(-1)^{n} A^{n}+\cdots,
$$

so we have

$$
U(t)=(I+A)^{-1} h(t) .
$$

Since $(I-A) h(t) \geq 0$, we obtain

$$
\begin{aligned}
U(t)= & (I-A) h(t)+A^{2}(I-A) h(t) \\
& +A^{4}(I-A) h(t) \cdots \geq 0 .
\end{aligned}
$$

Lemma 14. $(I-A) h(t) \leq 0$, and then the integral equation

$$
U(t)+b I_{0+}^{\alpha-\beta} U(t)+c I_{0+}^{\alpha} U(t)=h(t), \quad t \in I
$$

has one nonpositive solution.

\section{Constructive Existence of $(1,1)$-Solution of Two-Point Value Problem for the Fuzzy Linear Multiterm Fractional Differential Equation}

Let us consider the following fuzzy boundary value problem:

$$
\begin{aligned}
& \left({ }^{c} D_{1,1}^{\alpha} y\right)(t) \oplus b(t) \otimes\left({ }^{c} D_{1,1}^{\beta} y\right)(t) \oplus c(t) \otimes y(t) \\
& \quad=f(t), \quad t \in(0,1), \\
& y(0)=y_{0}, \\
& y(1)=y_{1},
\end{aligned}
$$

where $b, c \in C(I), b(t), c(t) \geq 0, y, f \in C\left(I, \mathbf{R}_{\mathrm{F}}\right), I=[0,1]$, $y_{0}, y_{1} \in \boldsymbol{R}_{F}, 1<\beta<\alpha \leq 2$.

Definition 15. A fuzzy valued function $y(t)$ is called a $(1,1)$ solution of the problem (38), (39) if it satisfies (38), (39) and ${ }^{c} D_{1,1}^{\alpha} y \in C\left(I, \mathbf{R}_{\mathrm{F}}\right)$.

By using r-cuts, we can obtain

$$
\begin{aligned}
& {\left[\left({ }^{c} D_{1,1}^{\alpha} y\right)(t) \oplus b(t) \otimes\left({ }^{c} D_{1,1}^{\beta} y\right)(t) \oplus c(t) \otimes y(t)\right]^{r}} \\
& \quad=[f(t)]^{r}, \quad r \in[0,1]
\end{aligned}
$$

and

$$
\begin{aligned}
& {\left[\left({ }^{c} D_{1,1}^{\alpha} y\right)(t)\right]^{r}+b(t)\left[\left({ }^{c} D_{1,1}^{\beta} y\right)(t)\right]^{r}} \\
& \quad+c(t)[y(t)]^{r}=[f(t)]^{r} . \\
& {\left[{ }^{c} D_{0+}^{\alpha} y_{1}(t, r),{ }^{c} D_{0+}^{\alpha} y_{2}(t, r)\right]} \\
& \quad+b(t)\left[{ }^{c} D_{0+}^{\beta} y_{1}(t, r),{ }^{c} D_{0+}^{\beta} y_{2}(t, r)\right] \\
& \quad+c(t)\left[y_{1}(t, r), y_{2}(t, r)\right]=\left[f_{1}(t, r), f_{2}(t, r)\right] .
\end{aligned}
$$


By the operation of intervals, we can have

$$
\begin{aligned}
& { }^{c} D_{0+}^{\alpha} y_{1}(t, r)+b(t){ }^{c} D_{0+}^{\beta} y_{1}(t, r)+c y_{1}(t, r) \\
& \quad=f_{1}(t, r), \\
& { }^{c} D_{0+}^{\alpha} y_{2}(t, r)+b(t){ }^{c} D_{0+}^{\beta} y_{2}(t, r)+c y_{2}(t, r) \\
& \quad=f_{2}(t, r) .
\end{aligned}
$$

In particular, the inequalities

$$
\begin{gathered}
y_{1}(t, r) \leq y_{2}(t, r), \\
{ }^{c} D_{0+}^{\alpha} y_{1}(t, r) \leq{ }^{c} D_{0+}^{\alpha} y_{2}(t, r), \\
{ }^{c} D_{0+}^{\beta} y_{1}(t, r) \leq{ }^{c} D_{0+}^{\beta} y_{2}(t, r)
\end{gathered}
$$

imply that $y_{1}(t, r)$ and $y_{2}(t, r)$ constitute interval and it is the same for ${ }^{c} D_{0+}^{\beta} y_{1}(t, r),{ }^{c} D_{0+}^{\beta} y_{2}(t, r)$ and ${ }^{c} D_{0+}^{\alpha} y_{1}(t, r)$, ${ }^{c} D_{0+}^{\alpha} y_{2}(t, r)$.

Let us denote the $r$-cut representation of $y_{0}, y_{1}$ by $\left[y_{0}\right]^{r}:=$ $\left[y_{0,1}(r), y_{0,2}(r)\right],\left[y_{1}\right]^{r}:=\left[y_{1,1}(r), y_{1,2}(r)\right]$. Then the cut forms of boundary conditions (39) are

$$
\begin{aligned}
& y_{1}(0, r)=y_{0,1}(r), \\
& y_{1}(1, r)=y_{1,1}(r), \\
& y_{2}(0, r)=y_{0,2}(r), \\
& y_{2}(1, r)=y_{1,2}(r) .
\end{aligned}
$$

Now the expression (42)-(44) is called cut problem of (38)-(39).

Definition 16. $\left(y_{1}(t, r), y_{2}(t, r)\right)$ is called a solution of (42)(44) if it satisfies (42)-(44) and ${ }^{c} D_{0+}^{\alpha} y_{1}(\cdot, r),{ }^{c} D_{0+}^{\alpha} y_{2}(\cdot, r) \in$ $C(I)$.

Theorem 17. (i) If $\left(y_{1}(t, r), y_{2}(t, r)\right)$ is a solution of the problem (42)-(44), then $\left(u_{1}(t, r), u_{2}(t, r)\right)$ satisfies

$$
\begin{aligned}
& u_{1}(t, r)+b(t) I_{0+}^{\alpha-\beta} u_{1}(t, r) \\
& \quad+c(t)\left((1-t) y_{0,1}(r)+t y_{1,1}(r)\right) \\
& \quad-c(t) \int_{0}^{1} G(t, s) u_{1}(s, r) d s=f_{1}(t, r), \\
& u_{2}(t, r)+b(t) I_{0+}^{\alpha-\beta} u_{2}(t, r) \\
& \quad+c(t)\left((1-t) y_{0,2}(r)+t y_{1,2}(r)\right) \\
& \quad-c(t) \int_{0}^{1} G(t, s) u_{2}(s, r) d s=f_{2}(t, r), \\
& u_{1}(t, r) \leq u_{2}(t, r), \\
& (1-t)\left(y_{0,2}(r)-y_{0,1}(r)\right)+t\left(y_{1,2}(r)-y_{1,1}(r)\right) \\
& \quad+I_{0+}^{\alpha}\left(u_{2}(t, r)-u_{1}(t, r)\right) \\
& \quad-\left.I_{0+}^{\alpha}\left(u_{2}(t, r)-u_{1}(t, r)\right)\right|_{t=1} \cdot t \geq 0,
\end{aligned}
$$

where ${ }^{c} D_{0+}^{\alpha} y_{1}(t, r)=u_{1}(t, r),{ }^{c} D_{0+}^{\alpha} y_{2}(t, r)=u_{2}(t, r)$. (ii) If $\left(u_{1}(t, r), u_{2}(t, r)\right)$ satisfies (45), then $\left(y_{1}(t, r), y_{2}(t, r)\right)$ is a solution of the problem (42)-(44), where

$$
\begin{array}{r}
y_{1}(t, r) \\
=(1-t) y_{0,1}(r)+t y_{1,1}(r) \\
\quad-\int_{0}^{1} G(t, s) u_{1}(s, r) d s, \\
y_{2}(t, r) \\
=(1-t) y_{0,2}(r)+t y_{1,2}(r) \\
\quad-\int_{0}^{1} G(t, s) u_{2}(s, r) d s,
\end{array}
$$

$G(t, s)$

$$
:=\frac{1}{\Gamma(\alpha)} \begin{cases}\frac{t}{(1-s)^{1-\alpha}}, & s \geq t, \\ \left(\frac{t}{(1-s)^{1-\alpha}}-\frac{1}{(t-s)^{1-\alpha}}\right), & s<t .\end{cases}
$$

Proof. Suppose $\left(y_{1}(t, r), y_{2}(t, r)\right)$ is a solution of the problem (42)-(44).

Then, we can get

$$
\begin{aligned}
& I_{0+}^{\alpha c} D_{0+}^{\alpha} y_{1}(t, r)=I_{0+}^{\alpha} u_{1}(t, r), \\
& I_{0+}^{\alpha c} D_{0+}^{\alpha} y_{2}(t, r)=I_{0+}^{\alpha} u_{2}(t, r) .
\end{aligned}
$$

From the boundary condition (44), we have

$$
\begin{aligned}
& y_{1}(t, r)=y_{0,1}(r)+c_{1} \cdot t+I_{0+}^{\alpha} u_{1}(t, r), \\
& y_{2}(t, r)=y_{0,2}(r)+d_{1} \cdot t+I_{0+}^{\alpha} u_{2}(t, r), \\
& y_{1}(1, r)=y_{0,1}(r)+c_{1}+\left.I_{0+}^{\alpha} u_{1}(t, r)\right|_{t=1}, \\
& y_{2}(1, r)=y_{0,2}(r)+d_{1}+\left.I_{0+}^{\alpha} u_{2}(t, r)\right|_{t=1}, \\
& y_{1,1}(r)=y_{0,1}(r)+c_{1}+\left.I_{0+}^{\alpha} u_{1}(t, r)\right|_{t=1}, \\
& y_{1,2}(r)=y_{0,2}(r)+d_{1}+\left.I_{0+}^{\alpha} u_{2}(t, r)\right|_{t=1}, \\
& y_{1,1}(r)-y_{0,1}(r)-\left.I_{0+}^{\alpha} u_{1}(t, r)\right|_{t=1}=c_{1}, \\
& y_{1,2}(r)-y_{0,2}(r)-\left.I_{0+}^{\alpha} u_{2}(t, r)\right|_{t=1}=d_{1} .
\end{aligned}
$$

Thus, we obtain

$$
\begin{aligned}
y_{1}(t, r)= & y_{0,1}(r) \\
& +\left(y_{1,1}(r)-y_{0,1}(r)-\left.I_{0+}^{\alpha} u_{1}(t, r)\right|_{t=1}\right) \\
& \cdot t+I_{0+}^{\alpha} u_{1}(t, r), \\
y_{2}(t, r)=y_{0,2}(r) & \\
& +\left(y_{1,2}(r)-y_{0,2}(r)-\left.I_{0+}^{\alpha} u_{2}(t, r)\right|_{t=1}\right) \\
& \cdot t+I_{0+}^{\alpha} u_{2}(t, r),
\end{aligned}
$$


and so we have

$$
\begin{aligned}
& { }^{c} D_{0+}^{\beta} y_{1}(t, r)=I_{0+}^{\alpha-\beta} u_{1}(t, r), \\
& { }^{c} D_{0+}^{\beta} y_{2}(t, r)=I_{0+}^{\alpha-\beta} u_{2}(t, r) .
\end{aligned}
$$

Using Green's function, (50) can be expressed as follows:

$$
\begin{aligned}
y_{1}(t, r)= & (1-t) y_{0,1}(r)+t y_{1,1}(r) \\
& -\int_{0}^{1} G(t, s) u_{1}(s, r) d s, \\
y_{2}(t, r)= & (1-t) y_{0,2}(r)+t y_{1,2}(r) \\
& -\int_{0}^{1} G(t, s) u_{2}(s, r) d s .
\end{aligned}
$$

From (43), (51), and (52), we can obtain (45). have

On the other hand, since ${ }^{c} D_{0+}^{\alpha} y_{1}(t, r) \leq{ }^{c} D_{0+}^{\alpha} y_{2}(t, r)$, we

$$
u_{1}(t, r) \leq u_{2}(t, r) \text {. }
$$

At last, from the inequality $y_{1}(t, r) \leq y_{2}(t, r)$, we can have

$$
\begin{aligned}
(1-t) & \left(y_{0,2}(r)-y_{0,1}(r)\right)+t\left(y_{1,2}(r)-y_{1,1}(r)\right) \\
+ & I_{0+}^{\alpha}\left(u_{2}(t, r)-u_{1}(t, r)\right) \\
- & \left.I_{0+}^{\alpha}\left(u_{2}(t, r)-u_{1}(t, r)\right)\right|_{t=1} \cdot t \geq 0 .
\end{aligned}
$$

Conversely, let us suppose that the pair $\left(u_{1}(t, r), u_{1}(t, r)\right)$ satisfies (45). From (46), we can get

$$
\begin{aligned}
& { }^{c} D_{0+}^{\alpha} y_{1}(t, r)=u_{1}(t, r), \\
& { }^{c} D_{0+}^{\alpha} y_{2}(t, r)=u_{2}(t, r) .
\end{aligned}
$$

Therefore, we have ${ }^{c} D_{0+}^{\alpha} y_{1}(t, r),{ }^{c} D_{0+}^{\alpha} y_{2}(t, r) \in C(I)$ and so we can get

$$
\begin{aligned}
& { }^{c} D_{0+}^{\beta} y_{1}(t, r)=I_{0+}^{\alpha-\beta} u_{1}(t, r), \\
& { }^{c} D_{0+}^{\beta} y_{2}(t, r)=I_{0+}^{\alpha-\beta} u_{2}(t, r),
\end{aligned}
$$

and

$$
\begin{aligned}
& { }^{c} D_{0+}^{\alpha} y_{1}(t, r)+b(t){ }^{c} D_{0+}^{\beta} y_{1}(t, r)+c(t) y_{1}(t, r) \\
& \quad=f_{1}(t, r), \\
& { }^{c} D_{0+}^{\alpha} y_{2}(t, r)+b(t){ }^{c} D_{0+}^{\beta} y_{2}(t, r)+c(t) y_{2}(t, r) \\
& \quad=f_{2}(t, r) .
\end{aligned}
$$

We can easily obtain the other inequalities and prove the boundary condition (44).

By this theorem, the existence of solutions of (42)-(44) is equivalent to the existence of solutions of (45).
Now, let us consider the integral equation

$$
\begin{aligned}
& u_{1}(t, r)+b(t) I_{0+}^{\alpha-\beta} u_{1}(t, r) \\
& \quad+c(t)\left((1-t) y_{0,1}(r)+t y_{1,1}(r)\right)+c(t) I_{0+}^{\alpha} u_{1}(t, r) \\
& \quad-\left.t c(t) I_{0+}^{\alpha} u_{1}(t, r)\right|_{t=1}=f_{1}(t, r), \\
& u_{2}(t, r)+b(t) I_{0+}^{\alpha-\beta} u_{2}(t, r) \\
& \quad+c(t)\left((1-t) y_{0,2}(r)+t y_{1,2}(r)\right)+c(t) I_{0+}^{\alpha} u_{2}(t, r) \\
& \quad-\left.t c(t) I_{0+}^{\alpha} u_{2}(t, r)\right|_{t=1}=f_{2}(t, r) .
\end{aligned}
$$

Assumption 1. $q:=(\|b\| / \Gamma(\alpha-\beta+1)+2\|c\| / \Gamma(\alpha+1))<1$.

Denote the following:

$$
\begin{aligned}
& g_{1}(t, r):=f_{1}(t, r)-c(t)\left((1-t) y_{0,1}(r)+t y_{1,1}(r)\right), \\
& g_{2}(t, r):=f_{2}(t, r)-c(t)\left((1-t) y_{0,2}(r)+t y_{1,2}(r)\right), \\
& u_{1}^{0}(t, r)=0, \\
& u_{2}^{0}(t, r)=0 .
\end{aligned}
$$

We are going to consider a the scheme of successive approximation

$$
\begin{aligned}
& u_{1}^{n+1}(t, r)+b(t) I_{0+}^{\alpha-\beta} u_{1}^{n+1}(t, r)+c(t) I_{0+}^{\alpha} u_{1}^{n+1}(t, r) \\
& \quad=g_{1}(t, r)+\left.t c(t) I_{0+}^{\alpha} u_{1}^{n}(t, r)\right|_{t=1}, \\
& u_{2}^{n+1}(t, r)+b(t) I_{0+}^{\alpha-\beta} u_{2}^{n+1}(t, r)+c(t) I_{0+}^{\alpha} u_{2}^{n+1}(t, r) \\
& \quad=g_{2}(t, r)+\left.t c(t) I_{0+}^{\alpha} u_{2}^{n}(t, r)\right|_{t=1} .
\end{aligned}
$$

Lemma 18. The sequence $\left(u_{1}^{n}(t, r), u_{2}^{n}(t, r)\right)$ that satisfies (60) is a Cauchy's sequence in $C(I) \times C(I)$.

Proof. We can have the following equations from $n^{\text {th }},(n+1)^{\text {th }}$ terms of the scheme of successive approximation

$$
\begin{aligned}
& \left(u_{1}^{n+1}(t, r)-u_{1}^{n}(t, r)\right) \\
& \quad+b(t) I_{0+}^{\alpha-\beta}\left(u_{1}^{n+1}(t, r)-u_{1}^{n}(t, r)\right) \\
& \quad+c(t) I_{0+}^{\alpha}\left(u_{1}^{n+1}(t, r)-u_{1}^{n}(t, r)\right) \\
& \quad=\left.t c(t) I_{0+}^{\alpha}\left(u_{1}^{n}(t, r)-u_{1}^{n-1}(t, r)\right)\right|_{t=1}, \\
& \left(u_{2}^{n+1}(t, r)-u_{2}^{n}(t, r)\right) \\
& \quad+b(t) I_{0+}^{\alpha-\beta}\left(u_{2}^{n+1}(t, r)-u_{2}^{n}(t, r)\right) \\
& \quad+c(t) I_{0+}^{\alpha}\left(u_{2}^{n+1}(t, r)-u_{2}^{n}(t, r)\right) \\
& \quad=\left.t c(t) I_{0+}^{\alpha}\left(u_{2}^{n}(t, r)-u_{2}^{n-1}(t, r)\right)\right|_{t=1} .
\end{aligned}
$$


For the first equation, we can get

$$
\begin{aligned}
& \left|u_{1}^{n+1}(t, r)-u_{1}^{n}(t, r)\right| \\
& \leq\|b\| I_{0+}^{\alpha-\beta}\left|u_{1}^{n+1}(t, r)-u_{1}^{n}(t, r)\right| \\
& \quad+\|c\| I_{0+}^{\alpha}\left|u_{1}^{n+1}(t, r)-u_{1}^{n}(t, r)\right| \\
& \quad+\|t c\| I_{0+}^{\alpha} \mid u_{1}^{n}(t, r)-u_{1}^{n-1}(t, r) \|_{t=1} \\
& \leq\left(\frac{\|b\|}{\Gamma(\alpha-\beta+1)}+\frac{\|c\|}{\Gamma(\alpha+1)}\right) \\
& \quad \cdot\left\|u_{1}^{n+1}(\cdot, r)-u_{1}^{n}(\cdot, r)\right\|+\frac{\|c\|}{\Gamma(\alpha+1)} \\
& \quad \cdot\left\|u_{1}^{n}(\cdot, r)-u_{1}^{n-1}(\cdot, r)\right\|
\end{aligned}
$$

and so we obtain

$$
\begin{aligned}
\left\|u_{1}^{n+1}(\cdot, r)-u_{1}^{n}(\cdot, r)\right\| \leq & \left(\frac{\|b\|}{\Gamma(\alpha-\beta+1)}+\frac{\|c\|}{\Gamma(\alpha+1)}\right) \\
& \cdot\left\|u_{1}^{n+1}(\cdot, r)-u_{1}^{n}(\cdot, r)\right\| \\
& +\frac{\|c\|}{\Gamma(\alpha+1)} \\
& \cdot\left\|u_{1}^{n}(\cdot, r)-u_{1}^{n-1}(\cdot, r)\right\| .
\end{aligned}
$$

If

$$
\gamma:=\frac{1}{1-q+\|c\| / \Gamma(\alpha+1)} \frac{\|c\|}{\Gamma(\alpha+1)}<1,
$$

we can get

$$
\begin{aligned}
\| & u_{1}^{n+1}(\cdot, r)-u_{1}^{n}(\cdot, r) \| \\
\leq & \frac{1}{1-(\|b\| / \Gamma(\alpha-\beta+1)+\|c\| / \Gamma(\alpha+1))} \frac{\|c\|}{\Gamma(\alpha+1)} \\
& \cdot\left\|u_{1}^{n}(\cdot, r)-u_{1}^{n-1}(\cdot, r)\right\| \\
= & \frac{1}{1-q+\|c\| / \Gamma(\alpha+1)} \frac{\|c\|}{\Gamma(\alpha+1)} \\
& \cdot\left\|u_{1}^{n}(\cdot, r)-u_{1}^{n-1}(\cdot, r)\right\| \\
\leq & \gamma\left\|u_{1}^{n}(\cdot, r)-u_{1}^{n-1}(\cdot, r)\right\| .
\end{aligned}
$$

As the same way, we can prove for $u_{2}(t, r)$.

Since the space $C(I)$ is complete, we can say that

$$
\begin{aligned}
& \exists \bar{u}_{1}(\cdot, r) \in C(I) ; \\
& u_{1}^{(n)}(\cdot, r) \longrightarrow \bar{u}_{1}(\cdot, r), \\
& \exists \bar{u}_{2}(\cdot, r) \in C(I) ; \\
& u_{2}^{(n)}(\cdot, r) \longrightarrow \bar{u}_{2}(\cdot, r) .
\end{aligned}
$$

Thus, we have

$$
\begin{gathered}
\bar{u}_{1}(t, r)+b(t) I_{0+}^{\alpha-\beta} \bar{u}_{1}(t, r)+c(t) I_{0+}^{\alpha} \bar{u}_{1}(t, r) \\
=g_{1}(t, r)+\left.t c(t) I_{0+}^{\alpha} \bar{u}_{1}(t, r)\right|_{t=1}, \\
\bar{u}_{2}(t, r)+b(t) I_{0+}^{\alpha-\beta} \bar{u}_{2}(t, r)+c(t) I_{0+}^{\alpha} \bar{u}_{2}(t, r) \\
=g_{2}(t, r)+\left.t c(t) I_{0+}^{\alpha} \bar{u}_{2}(t, r)\right|_{t=1} .
\end{gathered}
$$

Let us denote the following:

$$
\begin{aligned}
\operatorname{len}(g)(t, r):= & g_{2}(t, r)-g_{1}(t, r), \\
\operatorname{len}\left(u^{n}\right)(t, r):= & u_{2}^{n}(t, r)-u_{1}^{n}(t, r), \\
g_{1}(t, r):= & f_{1}(t, r) \\
& -c(t)\left((1-t) y_{0,1}(r)+t y_{1,1}(r)\right), \\
g_{2}(t, r):= & f_{2}(t, r) \\
& -c(t)\left((1-t) y_{0,2}(r)+t y_{1,2}(r)\right), \\
\operatorname{len}(g)(t, r)= & \operatorname{len}(f)(t, r) \\
& -c(t)\left((1-t) \operatorname{len}\left(y_{0}\right)+\operatorname{tlen}\left(y_{1}\right)\right) .
\end{aligned}
$$

Assumption 2. $(I-A)(\operatorname{len}(g)) \geq 0$.

Assumption 3. $(I-A)(t c(t)) \geq 0$.

Theorem 19. If $\bar{u}_{1}(t, r), \bar{u}_{2}(t, r)$ satisfy (67), then they satisfy

$$
\bar{u}_{1}(t, r) \leq \bar{u}_{2}(t, r), \quad t \in I .
$$

Proof. Let us consider a the scheme of successive approximation

$$
\begin{aligned}
& \operatorname{len}\left(u^{0}\right)(t, r)=0, \\
& \operatorname{len}\left(u^{n+1}\right)(t, r)+b(t) I_{0+}^{\alpha-\beta} \operatorname{len}\left(u^{n+1}\right)(t, r) \\
& \quad+c(t) I_{0+}^{\alpha} \operatorname{len}\left(u^{n+1}\right)(t, r)=\operatorname{len}(g)(t, r) \\
& \quad+\left.\operatorname{tc}(t) I_{0+}^{\alpha} \operatorname{len}\left(u^{n}\right)(t, r)\right|_{t=1} .
\end{aligned}
$$

When $n=0$, we can have the inequality $\operatorname{len}\left(u^{1}\right)(t, r) \geq 0$ from $\operatorname{len}\left(u^{0}\right)(t, r)=0$, Assumption 2, and Lemma 13 .

For any $n \geq 1$, let us suppose that $\operatorname{len}\left(u^{n}\right)(t, r) \geq 0$.

Then, we can obtain

$$
\begin{gathered}
(I-A)\left(\operatorname{len}(g)(t, r)+\left.t c(t) I_{0+}^{\alpha} \operatorname{len}\left(u^{n}\right)(t, r)\right|_{t=1}\right) \\
=(I-A)(\operatorname{len}(g))(t, r) \\
\quad+\left.I_{0+}^{\alpha} \operatorname{len}\left(u^{n}\right)(t, r)\right|_{t=1}(I-A)(t c(t)) \geq 0
\end{gathered}
$$

and so we have

$$
\operatorname{len}\left(u^{n+1}\right)(t, r) \geq 0 .
$$

By the limit of inequality (72), the proof is completed. 
Let us use the following notations:

$$
\begin{aligned}
\operatorname{len}\left(y_{0}\right)(t, r) & :=y_{0,2}(r)-y_{0,1}(r), \\
\operatorname{len}\left(y_{1}\right)(t, r) & :=y_{1,2}(r)-y_{1,1}(r), \\
\operatorname{len}(\bar{u})(t, r) & :=\bar{u}_{2}(t, r)-\bar{u}_{1}(t, r) .
\end{aligned}
$$

Assumption 4. len $(f)(t, r)-(\Gamma(\alpha+2)(b(t)+\Gamma(\alpha-\beta+1)) /(\Gamma(\alpha+$ 2) $\Gamma(\alpha-\beta+1)-\|c\| \Gamma(\alpha-\beta+1)-\|b\| \Gamma(\alpha+2)))\|\operatorname{len}(g)\| \geq 0$.

Theorem 20. $(1-t) \cdot \operatorname{len}\left(y_{0}\right)(t, r)+t \cdot \operatorname{len}\left(y_{1}\right)(t, r)+I_{0+}^{\alpha} \operatorname{len}(\bar{u})(t$, $r)-\left.I_{0+}^{\alpha} \operatorname{len}(\bar{u})(t, r)\right|_{t=1} \cdot t \geq 0$.

Proof. From equations (67), we can get

$$
\begin{aligned}
& \operatorname{len}(\bar{u})+b(t) I_{0+}^{\alpha-\beta} \operatorname{len}(\bar{u})+c(t) I_{0+}^{\alpha} \operatorname{len}(\bar{u}) \\
& =\operatorname{len}(g)+\left.t c(t) I_{0+}^{\alpha} \operatorname{len}(\bar{u})\right|_{t=1}, \\
& \operatorname{len}(g)(t, r) \\
& =\operatorname{len}(f)(t, r) \\
& \quad-c(t)\left((1-t) \operatorname{len}\left(y_{0}\right)+\operatorname{tlen}\left(y_{1}\right)\right) .
\end{aligned}
$$

Since $\operatorname{len}(\bar{u})$ is the solution of the equation

$$
\begin{aligned}
\operatorname{len}(\bar{u})= & \operatorname{len}(g)+\left.\operatorname{tc}(t) I_{0+}^{\alpha} \operatorname{len}(\bar{u})\right|_{t=1} \\
& -b(t) I_{0+}^{\alpha-\beta} \operatorname{len}(\bar{u})-c(t) I_{0+}^{\alpha} \operatorname{len}(\bar{u})
\end{aligned}
$$

and the inequality

$$
G(t, s) \leq \frac{s(1-s)^{\alpha-1}}{\Gamma(\alpha)},
$$

we have

$$
\begin{aligned}
\|\operatorname{len}(\bar{u})\| \leq & \|\operatorname{len}(g)\|+\|c\| \\
& \cdot\left\|\left.t I_{0+}^{\alpha} \operatorname{len}(\bar{u})\right|_{t=1}-I_{0+}^{\alpha} \operatorname{len}(\bar{u})\right\|+\|b\| \\
& \left\|I_{0+}^{\alpha-\beta} \operatorname{len}(\bar{u})\right\| \\
\leq & \|\operatorname{len}(g)\|+\|c\| \cdot \int_{0}^{1} \frac{s(1-s)^{\alpha-1}}{\Gamma(\alpha)} d s \\
& \cdot\|\operatorname{len}(\bar{u})\|+\frac{\|b\|}{\Gamma(\alpha-\beta+1)} \cdot\|\operatorname{len}(\bar{u})\| .
\end{aligned}
$$

We can get

$$
\int_{0}^{1} s(1-s)^{\alpha-1} d s=\frac{1}{\alpha+\alpha^{2}}
$$

and so we obtain

$$
\begin{gathered}
\|\operatorname{len}(\bar{u})\| \leq \\
+\operatorname{len}(g)\left\|+\frac{\|c\|}{\Gamma(\alpha+2)} \cdot\right\| \operatorname{len}(\bar{u}) \| \\
+\frac{\|b\|}{\Gamma(\alpha-\beta+1)} \cdot\|\operatorname{len}(\bar{u})\| .
\end{gathered}
$$

By Assumption 1, we can have the inequalities

$$
Q:=\frac{\|c\|}{\Gamma(\alpha+2)}+\frac{\|b\|}{\Gamma(\alpha-\beta+1)} \leq q<1,
$$

$\|\operatorname{len}(\bar{u})\| \leq \frac{1}{(1-Q)}\|\operatorname{len}(g)\|$.

We can get

$$
\begin{aligned}
c(t) & I_{0+}^{\alpha} \operatorname{len}(\bar{u})-\left.\operatorname{tc}(t) I_{0+}^{\alpha} \operatorname{len}(\bar{u})\right|_{t=1} \\
& =\operatorname{len}(g)-\operatorname{len}(\bar{u})-b(t) I_{0+}^{\alpha-\beta} \operatorname{len}(\bar{u})
\end{aligned}
$$

and since $c(t) \geq 0$, we have

$$
\begin{aligned}
& c(t)\left((1-t) \cdot \operatorname{len}\left(y_{0}\right)(t, r)+t \cdot \operatorname{len}\left(y_{1}\right)(t, r)\right. \\
&\left.\quad+I_{0+}^{\alpha} \operatorname{len}(\bar{u})(t, r)-\left.I_{0+}^{\alpha} \operatorname{len}(\bar{u})(t, r)\right|_{t=1} \cdot t\right) \geq 0
\end{aligned}
$$

and

$$
\begin{array}{r}
c(t)\left((1-t)-\operatorname{len}\left(y_{0}\right)(t, r)+t \cdot \operatorname{len}\left(y_{1}\right)(t, r)\right) \\
\quad+\operatorname{len}(g)-\operatorname{len}(\bar{u})-b(t) I_{0+}^{\alpha-\beta} \operatorname{len}(\bar{u}) \geq 0 .
\end{array}
$$

Since $c(t)\left((1-t) \cdot \operatorname{len}\left(y_{0}\right)(t, r)+t \cdot \operatorname{len}\left(y_{1}\right)(t, r)\right)+\operatorname{len}(g)=$ $\operatorname{len}(f)$, we have

$$
\operatorname{len}(f) \geq \operatorname{len}(\bar{u})+b(t) I_{0+}^{\alpha-\beta} \operatorname{len}(\bar{u}) .
$$

By equation (81), we have a condition

$$
\operatorname{len}(f) \geq \frac{\|\operatorname{len}(g)\|}{1-Q}\left(1+\frac{b(t)}{\Gamma(\alpha-\beta+1)}\right),
$$

that is,

$\operatorname{len}(f)(t, r)$

$$
\begin{aligned}
& -\frac{\Gamma(\alpha+2)(b(t)+\Gamma(\alpha-\beta+1))}{\Gamma(\alpha+2) \Gamma(\alpha-\beta+1)-\|c\| \Gamma(\alpha-\beta+1)-\|b\| \Gamma(\alpha+2)}\|\operatorname{len}(g)\| \\
& \geq 0 .
\end{aligned}
$$

Now let us consider if the solutions of (42)-(44) generate a fuzzy valued function and if the generated fuzzy valued function satisfies (38), (39).

From Theorem 17 and equation (43), we can have the following inequalities:

$$
\begin{gathered}
{ }^{c} D_{0+}^{\alpha} y_{1}(t, r) \leq{ }^{c} D_{0+}^{\alpha} y_{2}(t, r), \quad t \in I, r \in[0,1], \\
y_{1}(t, r) \leq y_{2}(t, r), \quad t \in I, r \in[0,1], \\
{ }^{c} D_{0+}^{\beta} y_{1}(t, r) \leq{ }^{c} D_{0+}^{\beta} y_{2}(t, r), \quad t \in I, r \in[0,1] .
\end{gathered}
$$

Hence, we can consider the sets of intervals

$$
\begin{aligned}
& \left\{U_{\alpha}(t, r):=\left[{ }^{c} D_{0+}^{\alpha} y_{1}(t, r),{ }^{c} D_{0+}^{\alpha} y_{2}(t, r)\right], r \in[0,1]\right\}, \\
& \left\{U_{\beta}(t, r):=\left[{ }^{c} D_{0+}^{\beta} y_{1}(t, r),{ }^{c} D_{0+}^{\beta} y_{2}(t, r)\right], r \in[0,1]\right\}, \\
& \left\{U_{0}(t, r):=\left[y_{1}(t, r), y_{2}(t, r)\right], r \in[0,1]\right\} .
\end{aligned}
$$


Theorem 21. The sets of intervals (89)-(91) generate continuous fuzzy valued functions.

Proof. Consider again the notion ${ }^{c} D_{0+}^{\alpha} y_{1}(t, r)=u_{1}(t, r)$, ${ }^{c} D_{0+}^{\alpha} y_{2}(t, r)=u_{2}(t, r)$.

Since $u_{1}(t, r), u_{2}(t, r)$ satisfy (45), then we can have $u_{2}(t, r) \geq u_{1}(t, r)$.

Let us prove $\left[u_{1}\left(t, r_{1}\right), u_{2}\left(t, r_{1}\right)\right] \supset\left[u_{1}\left(t, r_{2}\right), u_{2}\left(t, r_{2}\right)\right]$, where $0<r_{1}<r_{2} \leq 1$.

The proof is equivalent to

$$
\begin{aligned}
& u_{1}\left(t, r_{1}\right) \leq u_{1}\left(t, r_{2}\right), \\
& u_{2}\left(t, r_{2}\right) \leq u_{2}\left(t, r_{1}\right) .
\end{aligned}
$$

First, let us prove $u_{1}\left(t, r_{1}\right) \leq u_{1}\left(t, r_{2}\right)$. From the first equation of (45), we can have

$$
\begin{gathered}
u_{1}(t, r)+b(t) I_{0+}^{\alpha-\beta} u_{1}(t, r)+c(t) I_{0+}^{\alpha} u_{1}(t, r) \\
=g_{1}(t, r)+\left.t c(t) I_{0+}^{\alpha} u_{1}(t, r)\right|_{t=1}, \\
u_{2}(t, r)+b(t) I_{0+}^{\alpha-\beta} u_{2}(t, r)+c(t) I_{0+}^{\alpha} u_{2}(t, r) \\
=g_{2}(t, r)+\left.t c(t) I_{0+}^{\alpha} u_{2}(t, r)\right|_{t=1} .
\end{gathered}
$$

We need the following notions:

$$
\begin{aligned}
g_{1}(t, r) & :=f_{1}(t, r)-c(t)\left((1-t) y_{0,1}(r)+t y_{1,1}(r)\right), \\
g_{2}(t, r) & :=f_{2}(t, r)-c(t)\left((1-t) y_{0,2}(r)+t y_{1,2}(r)\right), \\
\Delta u_{1} & :=u_{1}\left(t, r_{2}\right)-u_{1}\left(t, r_{1}\right), \\
\Delta u_{2} & :=u_{2}\left(t, r_{2}\right)-u_{2}\left(t, r_{1}\right), \\
\Delta g_{1} & :=g_{1}\left(t, r_{2}\right)-g_{1}\left(t, r_{1}\right), \\
\Delta g_{2} & :=g_{2}\left(t, r_{2}\right)-g_{2}\left(t, r_{1}\right) .
\end{aligned}
$$

From (93), we can have

$$
\begin{gathered}
\Delta u_{1}+b(t) I_{0+}^{\alpha-\beta} \Delta u_{1}+c(t) I_{0+}^{\alpha} \Delta u_{1} \\
=\Delta g_{1}+\left.t c(t) I_{0+}^{\alpha} \Delta u_{1}\right|_{t=1} .
\end{gathered}
$$

Assumption 5. $\left((I-A) \Delta g_{1} \geq 0,(I-A) \Delta g_{2} \leq 0\right)$.

Let us consider a the scheme of successive approximation

$$
\begin{aligned}
& \Delta u_{1}{ }^{0}=0, \\
& \Delta u_{1}{ }^{n+1}+b(t) I_{0+}^{\alpha-\beta} \Delta u_{1}{ }^{n+1}+c(t) I_{0+}^{\alpha} \Delta u_{1}{ }^{n+1} \\
& \quad=\Delta g_{1}+\left.t c(t) I_{0+}^{\alpha} \Delta u_{1}{ }^{n}\right|_{t=1} .
\end{aligned}
$$

If $n=0$, then we can have

$$
\Delta u_{1}{ }^{1}+b(t) I_{0+}^{\alpha-\beta} \Delta u_{1}{ }^{1}+c(t) I_{0+}^{\alpha} \Delta u_{1}{ }^{1}=\Delta g_{1},
$$

and so from Assumption 5 and Lemma 13, we can obtain $\Delta u_{1}^{1} \geq 0$.

Suppose $\Delta u_{1}^{n} \geq 0$ for any $n \geq 1$.
Let us consider the equation

$$
\begin{aligned}
& \Delta u_{1}{ }^{n+1}+b(t) I_{0+}^{\alpha-\beta} \Delta u_{1}{ }^{n+1}+c(t) I_{0+}^{\alpha} \Delta u_{1}{ }^{n+1} \\
& =\Delta g_{1}+\left.t c(t) I_{0+}^{\alpha} \Delta u_{1}{ }^{n}\right|_{t=1} .
\end{aligned}
$$

By Assumptions 3, 5 and the induction, we can obtain ( $I-$ A) $\left(\Delta g_{1}+\left.t c(t) I_{0+}^{\alpha} \Delta u_{1}{ }^{(n)}\right|_{t=1}\right) \geq 0$ and so we have $\Delta u_{1}{ }^{(n+1)} \geq 0$ by Lemma 10 .

Finally, we have $\Delta u_{1} \geq 0$. We can also prove $\Delta u_{2} \leq 0$ as the same way by Lemma 14 .

Then, let us consider $u(t, r)=\bigcap_{n=1}^{\infty}\left[u_{1}\left(t, r_{k}\right), u_{2}\left(t, r_{k}\right)\right]$ for any nondecreasing sequence $r_{k} \in(0,1]$ which satisfies $\lim _{k \rightarrow \infty} r_{k}=r$, i.e., $\lim _{n \rightarrow \infty} u_{1}\left(t, r_{k}\right)=u_{1}(t, r)$, $\lim _{n \rightarrow \infty} u_{2}\left(t, r_{k}\right)=u_{2}(t, r)$.

From the first equation of (45), we have

$$
\begin{aligned}
& u_{1}(t, r)+b(t) I_{0+}^{\alpha-\beta} u_{1}(t, r) \\
& \quad+c(t)\left((1-t) y_{0,1}(r)+t y_{1,1}(r)\right) \\
& \quad+c(t) I_{0+}^{\alpha} u_{1}(t, r) \\
& \quad-\left.t c(t) I_{0+}^{\alpha} u_{1}(t, r)\right|_{t=1}=f_{1}(t, r), \\
& u_{1}\left(t, r_{k}\right)+b(t) I_{0+}^{\alpha-\beta} u_{1}\left(t, r_{k}\right) \\
& \quad+c(t)\left((1-t) y_{0,1}\left(r_{k}\right)+t y_{1,1}(r)\right) \\
& \quad+c(t) I_{0+}^{\alpha} u_{1}\left(t, r_{k}\right) \\
& \quad-\left.t c(t) I_{0+}^{\alpha} u_{1}\left(t, r_{k}\right)\right|_{t=1}=f_{1}\left(t, r_{k}\right) .
\end{aligned}
$$

and so we can get

$$
\begin{aligned}
u_{1}(t, r) & -u_{1}\left(t, r_{k}\right) \\
= & h\left(t, r_{k}, r\right)-b(t) I_{0+}^{\alpha-\beta}\left(u_{1}(t, r)-u_{1}\left(t, r_{k}\right)\right) \\
& -c(t) I_{0+}^{\alpha}\left(u_{1}(t, r)-u_{1}\left(t, r_{k}\right)\right) \\
& +\left.t c(t) I_{0+}^{\alpha}\left(u_{1}(t, r)-u_{1}\left(t, r_{k}\right)\right)\right|_{t=1} .
\end{aligned}
$$

Let us denote the following:

$$
\begin{aligned}
& \Delta_{k} u_{1}:=u_{1}(t, r)-u_{1}\left(t, r_{k}\right), \\
& h\left(t, r_{k}, r\right):=f_{1}(t, r)-f_{1}\left(t, r_{k}\right)-c(t) \\
& \quad \cdot\left((1-t)\left(y_{0,1}(r)-y_{0,1}\left(r_{k}\right)\right)\right. \\
& \left.\quad+t\left(y_{1,1}(r)-y_{1,1}\left(r_{k}\right)\right)\right) .
\end{aligned}
$$

Using the above notions, the equation can be written as

$$
\begin{gathered}
\Delta_{k} u_{1}+b(t) I_{0+}^{\alpha-\beta} \Delta_{k} u_{1}+c(t) I_{0+}^{\alpha} \Delta_{k} u_{1} \\
=h\left(t, r_{k}, r\right)+\left.t c(t) I_{0+}^{\alpha} \Delta_{k} u_{1}\right|_{t=1} .
\end{gathered}
$$

Now, we use the following scheme of successive approximation

$$
\begin{aligned}
& \Delta_{k} u_{1}{ }^{0}=0 \\
& \Delta_{k} u_{1}{ }^{n+1}+b(t) I_{0+}^{\alpha-\beta} \Delta_{k} u_{1}{ }^{n+1}+c(t) I_{0+}^{\alpha} \Delta_{k} u_{1}{ }^{n+1} \\
& \quad=h\left(t, r_{k}, r\right)+\left.t c(t) I_{0+}^{\alpha} \Delta_{k} u_{1}{ }^{n}\right|_{t=1} .
\end{aligned}
$$


When $n=0$, we have

$$
\begin{aligned}
& \Delta_{k} u_{1}{ }^{1}+b(t) I_{0+}^{\alpha-\beta} \Delta_{k} u_{1}{ }^{1}+c(t) I_{0+}^{\alpha} \Delta_{k} u_{1}{ }^{1} \\
& =h\left(t, r_{k}, r\right)
\end{aligned}
$$

and then from Lemma 12, we can obtain inequality $\left\|\Delta_{k} u_{1}{ }^{1}\right\| \leq$ $\left(e^{k_{*}} /(1-q)\right)\left\|h\left(\cdot, r_{k}, r\right)\right\|$.

Since $\lim _{r_{k} \rightarrow r}\left\|h\left(\cdot, r_{k}, r\right)\right\|=0$, we can get

$$
\lim _{r_{k} \longrightarrow r} \Delta_{k} u_{1}^{1}=0
$$

For any $n \in N$, suppose $\lim _{r_{k} \rightarrow r} \Delta_{k} u_{1}{ }^{n}=0$ and let us consider when $n+1$

We can have

$$
\begin{aligned}
& \Delta_{k} u_{1}{ }^{n+1}+b(t) I_{0+}^{\alpha-\beta} \Delta_{k} u_{1}^{n+1}+c(t) I_{0+}^{\alpha} \Delta_{k} u_{1}^{n+1} \\
& =h\left(t, r_{k}, r\right)+\left.t c(t) I_{0+}^{\alpha} \Delta_{k} u_{1}{ }^{n}\right|_{t=1},
\end{aligned}
$$

and so we can obtain

$$
\begin{aligned}
\lim _{r_{k} \rightarrow r} & \left(h\left(t, r_{k}, r\right)+\left.t c(t) I_{0+}^{\alpha} \Delta_{k} u_{1}{ }^{n}\right|_{t=1}\right) \\
= & \lim _{r_{k} \longrightarrow r} h\left(t, r_{k}, r\right)+\lim _{r_{k} \longrightarrow r}\left(\left.t c(t) I_{0+}^{\alpha} \Delta_{k} u_{1}{ }^{n}\right|_{t=1}\right) \\
= & \lim _{r_{k} \longrightarrow r} h\left(t, r_{k}, r\right) \\
& +t c(t) \lim _{r_{k} \longrightarrow r} \frac{1}{\Gamma(\alpha)} \int_{0}^{1} \frac{\Delta_{k} u_{1}^{n}\left(s, r_{k}\right)}{(1-s)^{1-\alpha}} d s \\
= & \lim _{r_{k} \longrightarrow r} h\left(t, r_{k}, r\right) \\
& +t c(t) \frac{1}{\Gamma(\alpha)} \int_{0}^{1} \lim _{r_{k} \longrightarrow r} \frac{\Delta_{k} u_{1}^{n}\left(s, r_{k}\right)}{(1-s)^{1-\alpha}} d s=0 .
\end{aligned}
$$

We can get

$$
\begin{aligned}
& \left\|\Delta_{k} u_{1}{ }^{n+1}\right\| \\
& \quad \leq \frac{e^{k_{*}}}{1-q} \max _{0 \leq t \leq 1}\left|h\left(t, r_{k}, r\right)+t c(t) I_{0+}^{\alpha} \Delta_{k} u_{1}{ }^{n}\right|_{t=1} \mid,
\end{aligned}
$$

and so we can have $\lim _{r_{k} \rightarrow r} \Delta_{k} u_{1}^{n+1}=0$.

Hence, we have $\lim _{r_{k} \rightarrow r} \Delta_{k} u_{1}=0$ and as the same way, we obtain $\lim _{r_{k} \rightarrow r} \Delta_{k} u_{2}=0$, where $\Delta_{k} u_{2}:=u_{2}(t, r)-u_{2}\left(t, r_{k}\right)$.

Finally, we proved that the set of intervals $\left\{\left[u_{1}(t, r), u_{2}(t, r)\right]\right\}_{r \in(0,1]}$ generates a fuzzy valued function.

Denote the fuzzy valued function generated by the set of intervals $\left\{\left[u_{1}(t, r), u_{2}(t, r)\right]\right\}_{r \in(0,1]}$ by a fuzzy valued function $\tilde{y}_{\alpha}(\mathrm{t})$.

For any $t_{*} \in I$, we can get

$$
\begin{aligned}
D & \left(\tilde{y}_{\alpha}(t), \tilde{y}_{\alpha}\left(t_{*}\right)\right) \\
& =\sup _{r \in[0,1]} \max \left\{\left|{ }^{c} D_{0+}^{\alpha} y_{1}(t, r)-{ }^{c} D_{0+}^{\alpha} y_{1}\left(t_{*}, r\right)\right|,\right. \\
& \left.\left|{ }^{c} D_{0+}^{\alpha} y_{2}(t, r)-{ }^{c} D_{0+}^{\alpha} y_{2}\left(t_{*}, r\right)\right|\right\} .
\end{aligned}
$$

Real-valued functions ${ }^{c} D_{0+}^{\alpha} y_{1}(t, r),{ }^{c} D_{0+}^{\alpha} y_{2}(t, r)$ are continuous since $y_{1}(t, r), y_{2}(t, r)$ are solutions of (42)-(44). So we have $D\left(\tilde{y}_{\alpha}(t), \tilde{y}_{\alpha}\left(t_{*}\right)\right) \longrightarrow 0\left(t \longrightarrow t_{*}\right)$.

Therefore, the fuzzy valued function $\tilde{y}_{\alpha}(t)$ is continuous.

As the same way, we can easily prove that the set of intervals $\left\{U_{\beta}(t, r)\right\}_{r}$ generates a fuzzy valued function.

Now let us consider that the set of intervals $\left\{U_{0}(t, r)\right\}_{r}$ generates a fuzzy valued function. The equation (46) can be expressed as follows:

$$
\begin{aligned}
y_{1}(t, r)= & (1-t) y_{0,1}(r)+t y_{1,1}(r) \\
& +\int_{0}^{1}(-G(t, s)) u_{1}(s, r) d s, \\
y_{2}(t, r)= & (1-t) y_{0,2}(r)+t y_{1,2}(r) \\
& +\int_{0}^{1}(-G(t, s)) u_{2}(s, r) d s .
\end{aligned}
$$

Because Green's function is continuous and the set of intervals $\left\{\left[u_{1}(t, r), u_{2}(t, r)\right]\right\}_{r \in(0,1]}$ generates a continuous fuzzy valued function, the set of intervals that is composed of right sides of these equations generates a fuzzy valued function.

Hence, the set of intervals $\left\{\left[y_{1}(t, r), y_{2}(t, r)\right]\right\}_{r}$ generates a fuzzy valued function.

Let us denote the fuzzy valued functions generated by (89), (90) by $\tilde{y}_{*}(t), \tilde{y}_{\beta}(t)$.

Then, we can easily prove that fuzzy valued functions $\tilde{y}_{*}(t), \tilde{y}_{\beta}(t)$ are continuous.

From equation (50), we can have

$$
\begin{array}{r}
y_{1}(t, r)+y_{0,1}(r) \cdot t+\left.I_{0+}^{\alpha} u_{1}(t, r)\right|_{t=1} \cdot t \\
=y_{0,1}(r)+y_{1,1}(r) \cdot t+I_{0+}^{\alpha} u_{1}(t, r), \\
y_{2}(t, r)+y_{0,2}(r) \cdot t+\left.I_{0+}^{\alpha} u_{2}(t, r)\right|_{t=1} \cdot t \\
=y_{0,2}(r)+y_{1,2}(r) \cdot t+I_{0+}^{\alpha} u_{2}(t, r),
\end{array}
$$

and we can get

$$
\left.\tilde{y}_{*} \oplus \tilde{y}_{0} \otimes t \oplus I_{0+}^{\alpha} \tilde{y}_{\alpha}\right|_{t=1} \otimes t=\tilde{y}_{0} \oplus \tilde{y}_{1} \otimes t \oplus I_{0+}^{\alpha} \tilde{y}_{\alpha} .
$$

Theorem 22. ${ }^{c} D_{1,1}^{\alpha} \tilde{y}_{*}=\tilde{y}_{\alpha},{ }^{c} D_{1,1}^{\beta} \tilde{y}_{*}=\tilde{y}_{\beta}$.

Proof. Let us consider again that the set of intervals,

$$
\begin{aligned}
& \left\{\left[{ }^{c} D_{0+}^{\alpha} I_{0+}^{\alpha} y_{1}(t, r),{ }^{c} D_{0+}^{\alpha} I_{0+}^{\alpha} y_{2}(t, r)\right]\right\}_{r} \\
& \quad=\left\{\left[y_{1}(t, r), y_{2}(t, r)\right]\right\}_{r}=\left\{U_{0}(t, r)\right\}_{r},
\end{aligned}
$$

generates a fuzzy valued function and the generated fuzzy valued function is $\widetilde{y}_{\alpha}$. So $I_{0+}^{\alpha} \widetilde{y}_{\alpha}$ is ${ }^{c}[(1,1)-\alpha]$-differentiable and ${ }^{c} D_{1,1}^{\alpha} I_{0+}^{\alpha} \tilde{y}_{\alpha}=\tilde{y}_{\alpha}$.

This shows that the left side of equation (112) is ${ }^{c}[(1,1)-\alpha]$-differentiable.

Then, we can have

$$
\begin{gathered}
{ }^{c} D_{1,1}^{\alpha} \tilde{y}_{*} \oplus{ }^{c} D_{1,1}^{\alpha}\left(\tilde{y}_{0} \otimes t\right) \oplus{ }^{c} D_{1,1}^{\alpha}\left(\left.I_{0+}^{\alpha} \tilde{y}_{\alpha}\right|_{t=1} \otimes t\right) \\
={ }^{c} D_{1,1}^{\alpha} \tilde{y}_{0} \oplus{ }^{c} D_{1,1}^{\alpha}\left(\tilde{y}_{1} \otimes t\right) \oplus{ }^{c} D_{1,1}^{\alpha} I_{0+}^{\alpha} \tilde{y}_{\alpha} .
\end{gathered}
$$


Furthermore, we can have ${ }^{c} D_{1,1}^{\alpha}\left(\tilde{y}_{0} \otimes t\right)=0$, ${ }^{c} D_{1,1}^{\alpha}\left(\left.I_{0+}^{\alpha} \tilde{y}_{\alpha}\right|_{t=1} \otimes t\right)=0$, so we obtain

$$
{ }^{c} D_{1,1}^{\alpha} \tilde{y}_{*}={ }^{c} D_{1,1}^{\alpha} I_{0+}^{\alpha} \tilde{y}_{\alpha}=\tilde{y}_{\alpha} .
$$

As the same way, we can prove that ${ }^{c} D_{1,1}^{\beta} \tilde{y}_{*}=\tilde{y}_{\beta}$.

Through the above investigations, we can see that $\tilde{y}_{*}$ is the solution of (38) and (39) by the sense of Definition 15.

In fact, from equation (112), we can see that

$$
\begin{aligned}
\tilde{y}_{*}= & \left(\tilde{y}_{0} \oplus \tilde{y}_{1} \otimes t \oplus I_{0+}^{\alpha} \tilde{y}_{\alpha}\right) \\
& \Theta_{H}\left(\left.\tilde{y}_{0} \otimes t \oplus I_{0+}^{\alpha} \tilde{y}_{\alpha}\right|_{t=1} \otimes t\right),
\end{aligned}
$$

and we can see that the boundary condition is concluded for the fuzzy valued function $\tilde{y}_{*}$.

At last, we have the fuzzy equation

$$
\tilde{y}_{\alpha}(t) \oplus b(t) \otimes \tilde{y}_{\beta}(t) \oplus c(t) \otimes \tilde{y}_{*}(t)=f(t) .
$$

Thus, the fuzzy valued function $\tilde{y}_{*}$ is a solution of the problem (38) and (39).

\section{The Numerical Solution of the Fuzzy Fractional Differential Equations by Using Haar Wavelet Operational Matrix}

Definition 23. The orthogonal set of Haar functions are defined in the interval $[0,1)$ by

$$
\begin{aligned}
& h_{0}(t)=\frac{1}{\sqrt{m}}, \\
& h_{i}(t)=\frac{1}{\sqrt{m}} \begin{cases}2^{j / 2}, & \frac{k-1}{2^{j}}<t \leq \frac{k-1 / 2}{2^{j}}, \\
2^{j / 2}, & \frac{k-1 / 2}{2^{j}}<t \leq \frac{k}{2^{j}}, \\
0, & \text { else, }\end{cases}
\end{aligned}
$$

where $i=0,1, \cdots m-1, m=2^{M}, M$ is a positive integer, and $j, k$ represent the integer decomposition of the index $i$.

We need the following notations:

$$
\begin{aligned}
H(t) & :=\left(h_{0}(t), h_{1}(t), \cdots, h_{m-1}(t)\right)^{T}, \\
C^{T} & :=\left(c_{0}, c_{1}, \cdots, c_{m-1}\right), \\
\Delta t & :=\frac{1}{m}=\frac{1}{2^{r}} \\
t_{k} & :=(k-0.5) \Delta t, \quad k=\overline{1, m} .
\end{aligned}
$$

The following matrix is called Haar wavelet matrix on the set of collocation points $\left\{t_{k}\right\}$ :

$$
H_{m a t}:=\left(\begin{array}{cccc}
h_{0}\left(t_{1}\right) & h_{0}\left(t_{2}\right) & \cdots & h_{0}\left(t_{m}\right) \\
h_{1}\left(t_{1}\right) & h_{1}\left(t_{2}\right) & \cdots & h_{1}\left(t_{m}\right) \\
\vdots & \vdots & \ddots & \vdots \\
h_{m-1}\left(t_{1}\right) & h_{m-1}\left(t_{2}\right) & \cdots & h_{m-1}\left(t_{m}\right)
\end{array}\right) .
$$

This is an orthogonal matrix; namely, $H_{m a t}^{-1}=H_{m a t}^{T}$. Put $\left(I_{0+}^{\alpha} H\right)(t):=\left(I_{0+}^{\alpha} h_{0}(t), I_{0+}^{\alpha} h_{1}(t), \cdots, I_{0+}^{\alpha} h_{m-1}(t)\right)^{T}$.

Definition 24. $m \times m$ matrix $F_{H}^{\alpha}$ determined by $\left(I_{0+}^{\alpha} H\right)(t) \approx$ $F_{H}^{\alpha} \cdot H(t)$ is called the operational matrix of the fractional integral of order $\alpha$.

If we use the matrix $F_{B}^{\alpha}$ determined by

$$
F_{B}^{\alpha}:=\frac{1}{m^{\alpha}} \frac{1}{\Gamma(\alpha+2)}\left(\begin{array}{ccccc}
1 & \xi_{1} & \xi_{2} & \cdots & \xi_{m-1} \\
0 & 1 & \xi_{1} & \cdots & \xi_{m-2} \\
0 & 0 & 1 & \cdots & \xi_{m-3} \\
\vdots & \vdots & \vdots & \ddots & \vdots \\
0 & 0 & \cdots & 0 & 1
\end{array}\right)
$$

the following equation holds:

$$
F_{H}^{\alpha}=H_{m a t} F_{B}^{\alpha} H_{m a t}^{T},
$$

where $\xi_{k}=(k+1)^{\alpha+1}-2 k^{\alpha+1}+(k-1)^{\alpha+1}$.

To solve the problem (38), (39), first, we need to solve the fractional integral equation sequence

$$
\begin{aligned}
& g_{1}(t, r):=f_{1}(t, r)-c(t)\left((1-t) y_{0,1}(r)+t y_{1,1}(r)\right), \\
& g_{2}(t, r):=f_{2}(t, r)-c(t)\left((1-t) y_{0,2}(r)+t y_{1,2}(r)\right), \\
& u_{1}^{0}(t, r)=0 \\
& u_{1}^{0}(t, r)=0 \\
& u_{1}^{n+1}(t, r)+b(t) I_{0+}^{\alpha-\beta} u_{1}^{n+1}(t, r)+c(t) I_{0+}^{\alpha} u_{1}^{n+1}(t, r) \\
& \quad=g_{1}(t, r)+\left.t c(t) I_{0+}^{\alpha} u_{1}^{n}(t, r)\right|_{t=1}, \\
& u_{2}^{n+1}(t, r)+b(t) I_{0+}^{\alpha-\beta} u_{2}^{n+1}(t, r)+c(t) I_{0+}^{\alpha} u_{2}^{n+1}(t, r) \\
& \quad=g_{2}(t, r)+\left.t c(t) I_{0+}^{\alpha} u_{2}^{n}(t, r)\right|_{t=1}
\end{aligned}
$$

by using operational matrix and get $u_{1}(t, r), u_{2}(t, r)$ approximately and then have $y_{1}(t, r), y_{2}(t, r)$ by the equations

$$
\begin{aligned}
y_{1}(t, r)= & y_{0,1}(r) \\
& +\left(y_{1,1}(r)-y_{0,1}(r)-\left.I_{0+}^{\alpha} u_{1}(t, r)\right|_{t=1}\right) \\
& \cdot t+I_{0+}^{\alpha} u_{1}(t, r), \\
y_{2}(t, r)= & y_{0,2}(r) \\
& +\left(y_{1,2}(r)-y_{0,2}(r)-\left.I_{0+}^{\alpha} u_{2}(t, r)\right|_{t=1}\right) \\
& \cdot t+I_{0+}^{\alpha} u_{2}(t, r) .
\end{aligned}
$$

We first calculate (124)

$$
\begin{aligned}
& C_{g_{1}}^{T}=\left(g_{1}\left(t_{1}, r\right), g_{1}\left(t_{2}, r\right), \cdots, g_{1}\left(t_{m}, r\right)\right) \cdot H_{m a t}^{T}, \\
& C_{g_{2}}^{T}=\left(g_{2}\left(t_{1}, r\right), g_{2}\left(t_{2}, r\right), \cdots, g_{2}\left(t_{m}, r\right)\right) \cdot H_{m a t}^{T},
\end{aligned}
$$


to solve (124) by using operational matrix. Using the notion

$$
\begin{aligned}
& u_{1}^{n}(t, r): \approx C_{u_{1}}^{n} H(t), \\
& u_{2}^{n}(t, r): \approx C_{u_{2}}^{n} H(t), \\
& g_{1}(t, r): \approx C_{g_{1}}^{T} H(t), \\
& g_{2}(t, r): \approx C_{g_{2}}^{T} H(t),
\end{aligned}
$$

we have the following repeat equations from (124).

$$
\begin{aligned}
& u_{1}^{n+1}(t, r)+b(t) I_{0+}^{\alpha-\beta} u_{1}^{n+1}(t, r)+c(t) I_{0+}^{\alpha} u_{1}^{n+1}(t, r) \\
& =g_{1}(t, r)+\left.t c(t) I_{0+}^{\alpha} u_{1}^{n}(t, r)\right|_{t=1}, \\
& u_{2}^{n+1}(t, r)+b(t) I_{0+}^{\alpha-\beta} u_{2}^{n+1}(t, r)+c(t) I_{0+}^{\alpha} u_{2}^{n+1}(t, r) \\
& =g_{2}(t, r)+\left.t c(t) I_{0+}^{\alpha} u_{2}^{n}(t, r)\right|_{t=1} . \\
& C_{u_{1}}^{n+1} H(t)+b(t) I_{0+}^{\alpha-\beta} C_{u_{1}}^{n+1} H(t)+c(t) I_{0+}^{\alpha} C_{u_{1}}^{n+1} H(t) \\
& =C_{g_{1}}^{T} H(t)+\left.t c(t) I_{0+}^{\alpha} C_{u_{1}}^{n} H(t)\right|_{t=1}, \\
& C_{u_{2}}^{n+1} H(t)+b(t) I_{0+}^{\alpha-\beta} C_{u_{2}}^{n+1} H(t)+c(t) I_{0+}^{\alpha} C_{u_{2}}^{n+1} H(t) \\
& =C_{g_{2}}^{T} H(t)+\left.t c(t) I_{0+}^{\alpha} C_{u_{2}}^{n} H(t)\right|_{t=1}, \\
& C_{u_{1}}^{n+1} H(t)+b(t) C_{u_{1}}^{n+1} I_{0+}^{\alpha-\beta} H(t)+c(t) C_{u_{1}}^{n+1} I_{0+}^{\alpha} H(t) \\
& =C_{g_{1}}^{T} H(t)+\left.t c(t) C_{u_{1}}^{n} I_{0+}^{\alpha} H(t)\right|_{t=1}, \\
& C_{u_{2}}^{n+1} H(t)+b(t) C_{u_{2}}^{n+1} I_{0+}^{\alpha-\beta} H(t)+c(t) C_{u_{2}}^{n+1} I_{0+}^{\alpha} H(t) \\
& =C_{g_{2}}^{T} H(t)+\left.t c(t) C_{u_{2}}^{n} I_{0+}^{\alpha} H(t)\right|_{t=1}, \\
& C_{u_{1}}^{n+1} H(t)+b(t) C_{u_{1}}^{n+1} F_{H}^{\alpha-\beta} H(t)+c(t) C_{u_{1}}^{n+1} F_{H}^{\alpha} H(t) \\
& =C_{g_{1}}^{T} H(t)+\left.t c(t) C_{u_{1}}^{n} F_{H}^{\alpha} H(t)\right|_{t=1}, \\
& C_{u_{2}}^{n+1} H(t)+b(t) C_{u_{2}}^{n+1} F_{H}^{\alpha-\beta} H(t)+c(t) C_{u_{2}}^{n+1} F_{H}^{\alpha} H(t) \\
& =C_{g_{2}}^{T} H(t)+\left.t c(t) C_{u_{2}}^{n} F_{H}^{\alpha} H(t)\right|_{t=1} .
\end{aligned}
$$

This is a point equation.

$$
\begin{array}{rl}
C_{u_{1}}^{n+1} & H\left(t_{k}\right)+b\left(t_{k}\right) C_{u_{1}}^{n+1} F_{H}^{\alpha-\beta} H\left(t_{k}\right) \\
& +c\left(t_{k}\right) C_{u_{1}}^{n+1} F_{H}^{\alpha} H\left(t_{k}\right) \\
= & C_{g_{1}}^{T} H\left(t_{k}\right)+t_{k} c\left(t_{k}\right) C_{u_{1}}^{n} F_{H}^{\alpha} H\left(t_{M}\right),
\end{array}
$$

$$
k=\overline{1, M} \text {. }
$$

On the other hand, we can have

$$
\begin{aligned}
C_{u_{1}}^{n+1} H\left(t_{k}\right)+b\left(t_{k}\right) C_{u_{1}}^{n+1} F_{H}^{\alpha-\beta} H\left(t_{k}\right) \\
\quad+c\left(t_{k}\right) C_{u_{1}}^{n+1} F_{H}^{\alpha} H\left(t_{k}\right) \\
=C_{u_{1}}^{n+1}\left(I+b\left(t_{k}\right) F_{H}^{\alpha-\beta}+c\left(t_{k}\right) F_{H}^{\alpha}\right) H\left(t_{k}\right) .
\end{aligned}
$$

Denote the following:

$$
\begin{aligned}
& A:=\left(\left(I+b\left(t_{1}\right) F_{H}^{\alpha-\beta}+c\left(t_{1}\right) F_{H}^{\alpha}\right)\right. \\
&\left.\cdot H\left(t_{1}\right), \cdots,\left(I+b\left(t_{M}\right) F_{H}^{\alpha-\beta}+c\left(t_{M}\right) F_{H}^{\alpha}\right) H\left(t_{M}\right)\right), \\
& B:=\left\{\begin{array}{l}
C_{g_{1}}^{T} H\left(t_{1}\right)+t_{1} c(t) C_{u_{1}}^{n} F_{H}^{\alpha} H\left(t_{M}\right) \\
C_{g_{1}}^{T} H\left(t_{2}\right)+t_{2} c(t) C_{u_{1}}^{n} F_{H}^{\alpha} H\left(t_{M}\right) \\
\vdots \\
C_{g_{1}}^{T} H\left(t_{M}\right)+t_{M} c(t) C_{u_{1}}^{n} F_{H}^{\alpha} H\left(t_{M}\right) .
\end{array}\right.
\end{aligned}
$$
follows:

Then, the simultaneous equation to determine $C_{u_{1}}^{n+1}$ is as

$$
A C_{u_{1}}^{n+1}=B
$$

Lemma 25. If $\|b\|_{C(I)}+\|c\|_{C(I)}<1$, equation (132) has a unique solution.

Proof. Prove that the coefficient matrix $A$ is nonnullity. We can have

$$
\begin{aligned}
A= & \left(\left(I+b\left(t_{1}\right) F_{H}^{\alpha-\beta}+c\left(t_{1}\right) F_{H}^{\alpha}\right)\right. \\
& \left.\cdot H\left(t_{1}\right), \cdots,\left(I+b\left(t_{M}\right) F_{H}^{\alpha-\beta}+c\left(t_{M}\right) F_{H}^{\alpha}\right) H\left(t_{M}\right)\right) \\
& =\left(H\left(t_{1}\right)+\left(b\left(t_{1}\right) F_{H}^{\alpha-\beta}+c\left(t_{1}\right) F_{H}^{\alpha}\right)\right. \\
& \cdot H\left(t_{1}\right), \cdots, H\left(t_{M}\right)+\left(b\left(t_{M}\right) F_{H}^{\alpha-\beta}+c\left(t_{M}\right) F_{H}^{\alpha}\right) \\
& \left.\cdot H\left(t_{M}\right)\right)=\left(H\left(t_{1}\right), \cdots, H\left(t_{M}\right)\right) \\
& +\left(\left(b\left(t_{1}\right) F_{H}^{\alpha-\beta}+c\left(t_{1}\right) F_{H}^{\alpha}\right)\right. \\
& \left.\cdot H\left(t_{1}\right), \cdots,\left(b\left(t_{M}\right) F_{H}^{\alpha-\beta}+c\left(t_{M}\right) F_{H}^{\alpha}\right) H\left(t_{M}\right)\right) \\
& =H_{m a t}+\widetilde{A}, \\
\widetilde{A} & :=\left(\left(b\left(t_{1}\right) F_{H}^{\alpha-\beta}+c\left(t_{1}\right) F_{H}^{\alpha}\right)\right. \\
& \left.\cdot H\left(t_{1}\right), \cdots,\left(b\left(t_{M}\right) F_{H}^{\alpha-\beta}+c\left(t_{M}\right) F_{H}^{\alpha}\right) H\left(t_{M}\right)\right), \\
A= & H_{m a t}+\widetilde{A}=\left(I+\widetilde{A} H_{m a t}^{T}\right) H_{m a t} .
\end{aligned}
$$

Thus, the nonnullity of the matrix $A$ is equivalent to the nonnullity of the matrix $\left(I+\widetilde{A} H_{\text {mat }}^{T}\right)$. On the other hand, the inequality $\left\|\widetilde{A} H_{\text {mat }}^{T}\right\|<1$ guarantees the nonnullity of the matrix $\left(I+\widetilde{A} H_{\text {mat }}^{T}\right)$.

Since the inequality

$$
\left\|\widetilde{A} H_{m a t}^{T}\right\|_{2} \leq\|\widetilde{A}\|_{2}\left\|H_{m a t}^{T}\right\|_{2}=\|\widetilde{A}\|_{2}
$$

holds, the inequality $\|\widetilde{A}\|_{2}<1$ is a sufficient condition to $\left\|\widetilde{A} H_{\text {mat }}^{T}\right\|<1$. And by the notion

$$
\begin{aligned}
\widetilde{A} & =\left(\left(b\left(t_{1}\right) F_{H}^{\alpha-\beta}+c\left(t_{1}\right) F_{H}^{\alpha}\right)\right. \\
& \left.\cdot H\left(t_{1}\right), \cdots,\left(b\left(t_{M}\right) F_{H}^{\alpha-\beta}+c\left(t_{M}\right) F_{H}^{\alpha}\right) H\left(t_{M}\right)\right),
\end{aligned}
$$


we can obtain

$$
\begin{aligned}
\widetilde{A}^{T} & =\left(\begin{array}{c}
H^{T}\left(t_{1}\right)\left(b\left(t_{1}\right)\left(F_{H}^{\alpha-\beta}\right)^{T}+c\left(t_{1}\right)\left(F_{H}^{\alpha}\right)^{T}\right) \\
H^{T}\left(t_{2}\right)\left(b\left(t_{2}\right)\left(F_{H}^{\alpha-\beta}\right)^{T}+c\left(t_{2}\right)\left(F_{H}^{\alpha}\right)^{T}\right) \\
\vdots \\
H^{T}\left(t_{M}\right)\left(b\left(t_{M}\right)\left(F_{H}^{\alpha-\beta}\right)^{T}+c\left(t_{M}\right)\left(F_{H}^{\alpha}\right)^{T}\right)
\end{array}\right) \\
= & \left(\begin{array}{c}
\left(b\left(t_{1}\right) H^{T}\left(t_{1}\right)\left(F_{H}^{\alpha-\beta}\right)^{T}+c\left(t_{1}\right) H^{T}\left(t_{1}\right)\left(F_{H}^{\alpha}\right)^{T}\right) \\
\left(b\left(t_{2}\right) H^{T}\left(t_{2}\right)\left(F_{H}^{\alpha-\beta}\right)^{T}+c\left(t_{2}\right) H^{T}\left(t_{2}\right)\left(F_{H}^{\alpha}\right)^{T}\right) \\
\vdots \\
\left(b\left(t_{M}\right) H^{T}\left(t_{M}\right)\left(F_{H}^{\alpha-\beta}\right)^{T}+c\left(t_{M}\right) H^{T}\left(t_{M}\right)\left(F_{H}^{\alpha}\right)^{T}\right)
\end{array}\right) .
\end{aligned}
$$

$$
D_{2}:=\left(\begin{array}{c}
c\left(t_{1}\right) H^{T}\left(t_{1}\right)\left(F_{H}^{\alpha}\right)^{T} \\
c\left(t_{2}\right) H^{T}\left(t_{2}\right)\left(F_{H}^{\alpha}\right)^{T} \\
\vdots \\
c\left(t_{M}\right) H^{T}\left(t_{M}\right)\left(F_{H}^{\alpha}\right)^{T}
\end{array}\right)
$$

we have

$$
\begin{aligned}
\widetilde{A}^{T} & =D_{1}+D_{2}, \\
\left\|\widetilde{A}^{T}\right\|_{2} & =\left\|D_{1}+D_{2}\right\|_{2} \leq\left\|D_{1}\right\|_{2}+\left\|D_{2}\right\|_{2} .
\end{aligned}
$$

Now let us consider that

By using the notion of

$$
D_{1}:=\left(\begin{array}{c}
b\left(t_{1}\right) H^{T}\left(t_{1}\right)\left(F_{H}^{\alpha-\beta}\right)^{T} \\
b\left(t_{2}\right) H^{T}\left(t_{2}\right)\left(F_{H}^{\alpha-\beta}\right)^{T} \\
\vdots \\
b\left(t_{M}\right) H^{T}\left(t_{M}\right)\left(F_{H}^{\alpha-\beta}\right)^{T}
\end{array}\right),
$$

$$
\left\|D_{1}\right\|_{2}:=\left\|\left(\begin{array}{c}
b\left(t_{1}\right) H^{T}\left(t_{1}\right)\left(F_{H}^{\alpha-\beta}\right)^{T} \\
b\left(t_{2}\right) H^{T}\left(t_{2}\right)\left(F_{H}^{\alpha-\beta}\right)^{T} \\
\vdots \\
b\left(t_{M}\right) H^{T}\left(t_{M}\right)\left(F_{H}^{\alpha-\beta}\right)^{T}
\end{array}\right)\right\|_{2}
$$

We already knew that

$$
\begin{gathered}
\left(\begin{array}{c}
b\left(t_{1}\right) H^{T}\left(t_{1}\right)\left(F_{H}^{\alpha-\beta}\right)^{T} \\
b\left(t_{2}\right) H^{T}\left(t_{2}\right)\left(F_{H}^{\alpha-\beta}\right)^{T} \\
\vdots \\
b\left(t_{M}\right) H^{T}\left(t_{M}\right)\left(F_{H}^{\alpha-\beta}\right)^{T}
\end{array}\right)\left(\begin{array}{c}
b\left(t_{1}\right) H^{T}\left(t_{1}\right)\left(F_{H}^{\alpha-\beta}\right)^{T} \\
b\left(t_{2}\right) H^{T}\left(t_{2}\right)\left(F_{H}^{\alpha-\beta}\right)^{T} \\
\vdots \\
b\left(t_{M}\right) H^{T}\left(t_{M}\right)\left(F_{H}^{\alpha-\beta}\right)^{T}
\end{array}\right)^{T} \\
=\left(\begin{array}{c}
b\left(t_{1}\right) H^{T}\left(t_{1}\right)\left(F_{H}^{\alpha-\beta}\right)^{T} \\
b\left(t_{2}\right) H^{T}\left(t_{2}\right)\left(F_{H}^{\alpha-\beta}\right)^{T} \\
\vdots \\
b\left(t_{M}\right) H^{T}\left(t_{M}\right)\left(F_{H}^{\alpha-\beta}\right)^{T}
\end{array}\right)\left(b\left(t_{1}\right) F_{H}^{\alpha-\beta} H\left(t_{1}\right), b\left(t_{2}\right) F_{H}^{\alpha-\beta} H\left(t_{2}\right), \cdots, b\left(t_{M}\right) F_{H}^{\alpha-\beta} H\left(t_{M}\right)\right)
\end{gathered}
$$

and because

$$
\begin{aligned}
& \left\langle b\left(t_{1}\right) H^{T}\left(t_{1}\right)\left(F_{H}^{\alpha-\beta}\right)^{T}, b\left(t_{1}\right) F_{H}^{\alpha-\beta} H\left(t_{1}\right)\right\rangle \\
& =\left(b\left(t_{1}\right)\right)^{2} H^{T}\left(t_{1}\right)\left(F_{H}^{\alpha-\beta}\right)^{T} F_{H}^{\alpha-\beta} H\left(t_{1}\right) \\
& =\left(b\left(t_{1}\right)\right)^{2}, \\
& \left\langle b\left(t_{1}\right) H^{T}\left(t_{1}\right)\left(F_{H}^{\alpha-\beta}\right)^{T}, b\left(t_{2}\right) F_{H}^{\alpha-\beta} H\left(t_{2}\right)\right\rangle \\
& =b\left(t_{1}\right) b\left(t_{2}\right) H^{T}\left(t_{1}\right)\left(F_{H}^{\alpha-\beta}\right)^{T} F_{H}^{\alpha-\beta} H\left(t_{2}\right)=0,
\end{aligned}
$$

we obtain

$$
\begin{gathered}
\left(\begin{array}{c}
b\left(t_{1}\right) H^{T}\left(t_{1}\right)\left(F_{H}^{\alpha-\beta}\right)^{T} \\
b\left(t_{2}\right) H^{T}\left(t_{2}\right)\left(F_{H}^{\alpha-\beta}\right)^{T} \\
\vdots \\
b\left(t_{M}\right) H^{T}\left(t_{M}\right)\left(F_{H}^{\alpha-\beta}\right)^{T}
\end{array}\right)\left(\begin{array}{c}
b\left(t_{1}\right) H^{T}\left(t_{1}\right)\left(F_{H}^{\alpha-\beta}\right)^{T} \\
b\left(t_{2}\right) H^{T}\left(t_{2}\right)\left(F_{H}^{\alpha-\beta}\right)^{T} \\
\vdots \\
b\left(t_{M}\right) H^{T}\left(t_{M}\right)\left(F_{H}^{\alpha-\beta}\right)^{T}
\end{array}\right)^{T} \\
=\operatorname{diag}\left(\left(b\left(t_{1}\right)\right)^{2},\left(b\left(t_{2}\right)\right)^{2}, \cdots,\left(b\left(t_{M}\right)\right)^{2}\right) .
\end{gathered}
$$

Thus, we have

$$
\left\|D_{1}\right\|_{2} \leq \sqrt{\max _{k}\left(b\left(t_{k}\right)\right)^{2}} \leq\|b\|_{C(I)} .
$$


As the same way, we can obtain

$$
\left\|D_{2}\right\|_{2} \leq \sqrt{\max _{k}\left(c\left(t_{k}\right)\right)^{2}} \leq\|c\|_{C(I)} .
$$

Finally, we can see that

$$
\|\widetilde{A}\|_{2}=\left\|\widetilde{A}^{T}\right\|_{2} \leq\|b\|_{C(I)}+\|c\|_{C(I)} .
$$

We compute the solutions $u_{1}(t, r), u_{2}(t, r)$ of (124) by using the limit $C_{u_{1}}^{T}, C_{u_{2}}^{T}$ in the repeat equation (132).

Now, we consider the algorithm to compute (125) by using the operational matrix. We can rewrite the equation

$$
\begin{aligned}
y_{1}(t, r)= & y_{0,1}(r) \\
& +\left(y_{1,1}(r)-y_{0,1}(r)-\left.I_{0+}^{\alpha} u_{1}(t, r)\right|_{t=1}\right) \\
& \cdot t+I_{0+}^{\alpha} u_{1}(t, r) \\
y_{2}(t, r)= & y_{0,2}(r) \\
& +\left(y_{1,2}(r)-y_{0,2}(r)-\left.I_{0+}^{\alpha} u_{2}(t, r)\right|_{t=1}\right) \\
& \cdot t+I_{0+}^{\alpha} u_{2}(t, r)
\end{aligned}
$$

by using the notion

$$
\begin{aligned}
& h_{1}(t, r)=(1-t) y_{0,1}(r)+t y_{1,1}(r) \\
& h_{2}(t, r)=(1-t) y_{0,2}(r)+t y_{1,2}(r)
\end{aligned}
$$

as follows:

$$
\begin{aligned}
& y_{1}(t, r)=h_{1}(t, r)-\left.I_{0+}^{\alpha} u_{1}(t, r)\right|_{t=1} \cdot t+I_{0+}^{\alpha} u_{1}(t, r) \\
& y_{2}(t, r)=h_{2}(t, r)-\left.I_{0+}^{\alpha} u_{2}(t, r)\right|_{t=1} \cdot t+I_{0+}^{\alpha} u_{2}(t, r) .
\end{aligned}
$$

First, we denote the following as

$$
\begin{aligned}
& h_{1}(t, r): \approx C_{h_{1}}^{T} H(t), \\
& h_{2}(t, r): \approx C_{h_{2}}^{T} H(t)
\end{aligned}
$$

and compute

$$
\begin{aligned}
& C_{h_{1}}^{T}=\left(h_{1}\left(t_{1}, r\right), h_{1}\left(t_{2}, r\right), \cdots, h_{1}\left(t_{m}, r\right)\right) \cdot H_{m a t}^{T}, \\
& C_{h_{2}}^{T}=\left(h_{2}\left(t_{1}, r\right), h_{2}\left(t_{2}, r\right), \cdots, h_{2}\left(t_{m}, r\right)\right) \cdot H_{\text {mat }}^{T} .
\end{aligned}
$$

Then, $y_{1}(t, r), y_{2}(t, r)$ are computed by

$$
\begin{aligned}
& y_{1}(t, r) \approx C_{h_{1}}^{T} H(t)-t C_{u_{1}}^{T} F_{H}^{\alpha} H\left(t_{M}\right)+C_{u_{1}}^{T} F_{H}^{\alpha} H(t) \\
& y_{2}(t, r) \approx C_{h_{2}}^{T} H(t)-t C_{u_{2}}^{T} F_{H}^{\alpha} H\left(t_{M}\right)+C_{u_{2}}^{T} F_{H}^{\alpha} H(t)
\end{aligned}
$$

from (132).

\section{Numerical Examples}

Example 1. Consider a FVBP

$$
\begin{aligned}
& D_{1,1}^{(2)} y(t) \oplus b \otimes\left({ }^{c} D_{1,1}^{1.5} y\right)(t) \oplus c \otimes y(t) \\
& \quad=(1+t) \otimes(7.9,8,8.1), \quad t \in(0,1), \\
& y(0)=(-0.1,0,0.1), \\
& y(1)=(-0.1,0,0.1),
\end{aligned}
$$

where $b, c \in \mathbf{R}_{+}, y \in C\left([0,1], \mathbf{R}_{\mathrm{F}}\right)$ and the boundary values $(-0.1,0,0.1),(-0.1,0,0.1)$, and $(7.9,8,8.1)$ are triangular fuzzy numbers. Now, let us consider the conditions for Assumptions 1-5.

A condition for Assumption 1:

$$
\begin{aligned}
q & :=\left(\frac{\|b\|}{\Gamma(\alpha-\beta+1)}+\frac{2\|c\|}{\Gamma(\alpha+1)}\right)=1.12838 b+c \\
& <1
\end{aligned}
$$

A condition for Assumption 2: we can have

$$
\begin{aligned}
\operatorname{len}(f)(t, r) & =f_{2}(t, r)-f_{1}(t, r) \\
& =0.2(1-r)(1+t), \\
\operatorname{len}(g)(t, r) & =0.2(1-r)(1+t)-c 0.2(1-r) \\
& =0.2(1-r)(1+t-c)
\end{aligned}
$$

and so we can obtain an inequality

$$
(I-A)(1+t-c) \geq 0
$$

for a condition for Assumption 2.

Consequently, we can have

$$
\begin{aligned}
& (I-A)(1+t-c)=(1+t-c)-b I_{0+}^{\alpha-\beta}(1+t-c) \\
& -c I_{0+}^{\alpha}(1+t-c)=(1+t-c) \\
& -\frac{b}{\Gamma(1.5)}\left((1-c) t^{0.5}+\frac{t^{1.5}}{1.5}\right) \\
& -\frac{c}{2}\left((1-c) t^{2}+\frac{t^{3}}{3}\right) \geq(1-c) \\
& -\left(1.12838 b\left((1-c)+\frac{1}{1.5}\right)-\frac{c}{2}\left((1-c)+\frac{1}{3}\right)\right) \\
& =0.5(-2.54858+c)(-0.78475+c) \\
& +b(-1.88063+1.12838 c)
\end{aligned}
$$

and so the condition for Assumption 2 is

$$
\begin{aligned}
& 0.5(-2.54858+c)(-0.78475+c) \\
& +b(-1.88063+1.12838 c) \geq 0 .
\end{aligned}
$$


A condition for Assumption 3: we can easily prove that the equation

$$
\begin{aligned}
(I-A)(t) & =t-\frac{t^{\alpha-\beta}}{(\alpha-\beta)(\alpha-\beta+1)}-\frac{t^{\alpha}}{\alpha(\alpha+1)} \\
& =t-\frac{4 t^{0.5}}{3}-\frac{t^{2}}{6}
\end{aligned}
$$

is nonnegative.

A condition for Assumption 4: we can have

$$
\begin{aligned}
& \frac{\Gamma(\alpha+2)(b+\Gamma(\alpha-\beta+1))}{\Gamma(\alpha+2) \Gamma(\alpha-\beta+1)-\|c\| \Gamma(\alpha-\beta+1)-\|b\| \Gamma(\alpha+2)} \\
& \quad=\frac{6(b+0.886227)}{5.31736-6 b-0.886227 c} \\
& 0.2(1-r)(1+t) \\
& \quad-\frac{6(b+0.886227)}{5.31736-6 b-0.886227 c} 0.2(1-r)(2-c) \\
& \quad=0.2(1-r)\left((1+t)-\frac{6(b+0.886227)(2-c)}{5.31736-6 b-0.886227 c}\right) \\
& \quad \geq 0.2(1-r)\left(1-\frac{6(b+0.886227)(2-c)}{5.31736-6 b-0.886227 c}\right) .
\end{aligned}
$$

So, we can see that the inequality

$$
1-\frac{6(b+0.886227)(2-c)}{5.31736-6 b-0.886227 c} \geq 0
$$

is a sufficient condition for Assumption 4. Finally, we have

$$
5.31736+18 b-4.431135 c-6 b c \leq 0 .
$$

A condition for Assumption 5: first, we can have

$$
\begin{aligned}
f_{1}\left(t, r_{2}\right)-f_{1}\left(t, r_{1}\right)= & (1+t)\left(7.9+0.1 r_{2}\right) \\
& -(1+t)\left(7.9+0.1 r_{1}\right) \\
= & 0.1\left(r_{2}-r_{1}\right)(1+t), \\
y_{0,1}\left(r_{2}\right)-y_{0,1}\left(r_{1}\right)= & 0.1 r_{2}-0.1-\left(0.1 r_{1}-0.1\right) \\
= & 0.1\left(r_{2}-r_{1}\right), \\
y_{1,1}\left(r_{2}\right)-y_{1,1}\left(r_{1}\right)= & 0.1\left(r_{2}-r_{1}\right), \\
\Delta g_{1}= & 0.1\left(r_{2}-r_{1}\right)(1+t)(1-c) .
\end{aligned}
$$
0.

We can easily obtain $1-3 b / \sqrt{\pi}-1.5 c \geq 0$ for $(I-A) \Delta g_{1} \geq$

As the same way, we can have the same inequality for (IA) $\Delta g_{2} \leq 0$.

The following inequalities are conditions for the FBVP to have a solution.

(1) $1.12838 b+c<1$

(2) $1-c \geq 0$

(3) $0.5(-2.54858+c)(-0.78475+c)+b(-1.88063+$ $1.12838 c) \geq 0$

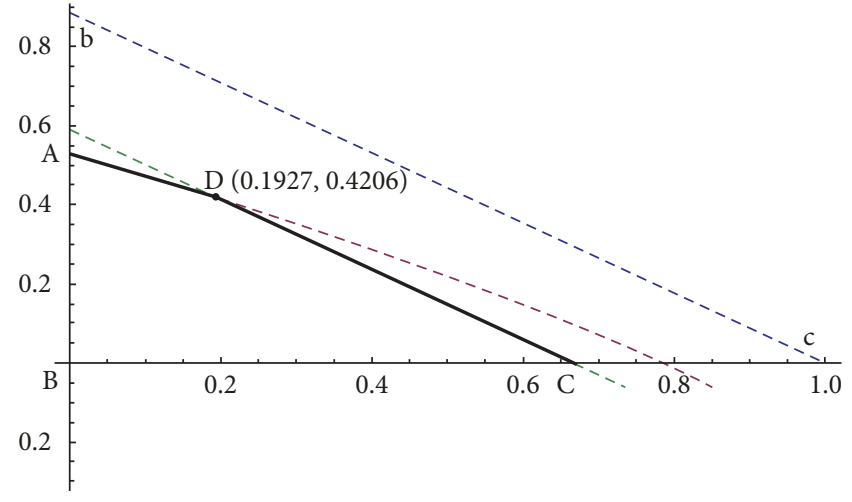

Figure 1: Area of $b$ and $c$ satisfying (1)-(5) for Example 1.

(4) $5.31736+18 b-4.431135 c-6 b c \leq 0$

(5) $1-3 b / \sqrt{\pi}-1.5 c \geq 0$

We show the area of $b, c$ that satisfies the above conditions on Figure 1.

Example 2. Consider the following fuzzy boundary value problem:

$$
\begin{aligned}
& D_{1,1}^{(2)} y(t) \oplus b(t) \otimes\left({ }^{c} D_{1,1}^{1.5} y\right)(t) \oplus c(t) \otimes y(t) \\
& \quad=(1+t) \otimes(7.9,8,8.1), \quad t \in(0,1), \\
& y(0)=(-0.1,0,0.1), \\
& y(1)=(-0.1,0,0.1),
\end{aligned}
$$

where $b, c \in C(I), y \in C\left([0,1], \mathbf{R}_{\mathrm{F}}\right), b(t), c(t) \geq 0$ and $(-0.1,0,0.1)$ and $(7.9,8,8.1)$ are triangular fuzzy numbers.

Assumption 1. $q:=(\|b\| / \Gamma(\alpha-\beta+1)+2\|c\| / \Gamma(\alpha+1))=$ $1.12838\|b\|+\|c\|<1$.

\section{Assumption 2.}

$$
\begin{aligned}
& \operatorname{len}(f)(t, r)=f_{2}(t, r)-f_{1}(t, r) \\
& =0.2(1-r)(1+t) \text {, } \\
& c(t)\left((1-t) \operatorname{len}\left(y_{0}\right)+t \operatorname{len}\left(y_{1}\right)\right)=c(t) 0.2(1-r), \\
& \operatorname{len}(g)(t, r)=0.2(1-r)(1+t)-c(t) 0.2(1-r) \\
& =0.2(1-r)(1+t-c(t)) \text {. }
\end{aligned}
$$

We can have $(I-A)(1+t-c(t)) \geq 0$ as a condition for Assumption 2.

Assumption 3. $(I-A)(t c(t)) \geq 0$. 
Assumption 4. Denote $\left|\left\|c\left|\|:=\min _{t \in[0,1]}\right| c(t) \mid\right.\right.$; then we get

$$
\begin{gathered}
0.2(1-r)(1+t)-\frac{6(b(t)+0.886227)}{5.31736-6\|b\|-0.886227\|c\|} \\
\cdot 0.2(1-r)(2-\||| c|| \mid)=0.2(1-r) \\
\cdot\left((1+t)-\frac{6(b(t)+0.886227)(2-\||| c|| \mid)}{5.31736-6\|b\|-0.886227\|c\|}\right) \\
\geq 0.2(1-r) \\
\cdot\left(1-\frac{6(b(t)+0.886227)(2-\||| c|| \mid)}{5.31736-6\|b\|-0.886227\|c\|}\right) .
\end{gathered}
$$

So we obtain

$$
1 \geq \frac{6(b(t)+0.886227)(2-\||\| c| \mid)}{5.31736-6\|b\|-0.886227\|c\|} .
$$

Assumption 5. We can obtain $1-\|c\| \geq 0,1-3\|b\| / \sqrt{\pi}-$ $1.5\|c\| \geq 0$ as Example 1 .

Sufficient conditions are given as follows:

(1) $1.12838\|b\|+\|c\|<1$

(2) $(I-A)(1+t-c(t)) \geq 0$

(3) $(I-A)(t c(t)) \geq 0$

(4) $1 \geq 6(b(t)+0.886227)(2-\||| c|| \mid) /(5.31736-6\|b\|-$ $0.886227\|c\|)$

(5) $1-3\|b\| / \sqrt{\pi}-1.5\|c\| \geq 0$

Let us consider especially for $c(t)=0.5 t /\left(1+t^{2}\right), b(t)=$ $0.2(1-t)$. We can obtain that

$$
\begin{aligned}
\|c\| & =0.25, \\
\|b\| & =0.2, \\
\||c|\| & =0 .
\end{aligned}
$$

$$
\begin{aligned}
1.12838\|b\|+\|c\| & =1.12838 \cdot 0.2+0.25=0.475676 \\
& <1,
\end{aligned}
$$

That is, condition 1 holds. Using the inequalities $0.75+t \leq$ $c(t) \leq 1+0.75 t$, we can have

$$
\begin{aligned}
& (I-A)(1+t-c(t)) \\
& =(1+t-c(t))-A(1+t-c(t)) \\
& =1+t-\frac{0.5 t}{\left(1+t^{2}\right)} \\
& \quad-0.2(1-t) I_{0+}^{\alpha-\beta}\left(1+t-\frac{0.5 t}{\left(1+t^{2}\right)}\right) \\
& \quad-\frac{0.5 t}{\left(1+t^{2}\right)} I_{0+}^{\alpha}\left(1+t-\frac{0.5 t}{\left(1+t^{2}\right)}\right)
\end{aligned}
$$

$$
\begin{aligned}
& \geq t+0.75-0.2(1-t) I_{0+}^{0.5}(1+0.75 t) \\
& \quad-\frac{0.5 t}{\left(1+t^{2}\right)} I_{0+}^{2}(1+0.75 t) \\
& =t+0.75-\frac{0.2(1-t)\left(2 t^{0.5}+t^{1.5}\right)}{\Gamma(0.5)} \\
& \quad-\frac{0.5 t}{\left(1+t^{2}\right)} \frac{0.5 t^{2}+0.125 t^{3}}{\Gamma(2)}, \\
& \geq t+0.75-0.1\left(2 t^{0.5}+t^{1.5}\right) \\
& \quad-(1+0.75 t)\left(0.5 t^{2}+0.125 t^{3}\right) \\
& \geq t+0.55-0.1 t^{1.5} \\
& \quad-(1+0.75 t)\left(0.5 t^{2}+0.125 t^{3}\right) \geq 0,
\end{aligned}
$$

i.e., condition 3 holds.

We can obtain

$$
\begin{aligned}
(I-A)(t c(t))= & t c(t)-A(t c(t)) \\
= & \frac{0.5 t^{2}}{\left(1+t^{2}\right)}-A\left(\frac{0.5 t^{2}}{\left(1+t^{2}\right)}\right) \\
= & \frac{0.5 t^{2}}{\left(1+t^{2}\right)}+0.01 t I_{0+}^{\alpha-\beta}\left(\frac{t^{2}}{\left(1+t^{2}\right)}\right) \\
& -0.01 I_{0+}^{\alpha-\beta}\left(\frac{t^{2}}{\left(1+t^{2}\right)}\right) \\
& -\frac{0.25 t}{\left(1+t^{2}\right) I_{0+}^{\alpha}\left(t^{2} /\left(1+t^{2}\right)\right)} \\
\geq & \frac{0.5 t^{2}}{\left(1+t^{2}\right)}+0.005 t I_{0+}^{\alpha-\beta} t^{2} \\
& -0.01 I_{0+}^{\alpha-\beta} t^{2}-\frac{0.25 t}{\left(1+t^{2}\right) I_{0+}^{\alpha} t^{2}} \\
& +\frac{0.5 t^{2}}{1+t^{2}}-\frac{0.0208333 t^{5}}{1+t^{2}} \geq 0 \\
= & \frac{0.5 t^{2}}{\left(1+t^{2}\right)}+0.005 \cdot 0.601802 t^{3.5} \\
& -0.01 \cdot 0.601802 t^{2.5}-\frac{0.25 t^{5}}{12\left(1+t^{2}\right)} \\
& 0.00601802 t^{2.5}+0.00300901 t^{3.5} \\
& \\
& \\
& \\
& \\
& \\
& \\
& \\
&
\end{aligned}
$$

and since $1-3\|b\| / \sqrt{\pi}-1.5\|c\|=1-0.338514-0.375=$ $0.286486>0$, condition 5 holds.

Through the investigations, we can see that the problem of Example 2 has a solution when $c(t)=0.5 t /\left(1+t^{2}\right), b(t)=$ 
TABLE 1: In case of $r=1$ for Example 2.

\begin{tabular}{lllll}
\hline$t$ & $y_{1}(t, 1)$ & $y_{2}(t, 1)$ & $y_{1}^{\prime \prime}(t, 1)$ & $y_{2}^{\prime \prime}(t, 1)$ \\
\hline 0.015025 & -0.104378 & -0.104378 & 8.04222 & 9.04222 \\
0.140625 & -0.867268 & -0.867268 & 12.0982 & 9.80614 \\
0.265625 & -1.47628 & -1.47628 & 14.7463 & 12.0982 \\
0.390625 & -1.89576 & -1.89576 & 17.7042 & 17.7042 \\
0.515625 & -2.08441 & -2.08441 & 20.9508 & 20.9508 \\
0.640625 & -1.99603 & -1.99603 & 24.4836 & 24.4836 \\
0.765625 & -1.5799 & -1.5799 & 28.3149 & 28.3149 \\
0.890625 & -0.780805 & -0.780805 & 31.3979 & 31.3979 \\
0.984375 & 0.105687 & 0.105687 & & \\
\hline
\end{tabular}

TABLE 2: In case of $r=0.5$ for Example 2.

\begin{tabular}{lcccc}
\hline$t$ & $y_{1}(t, 1)$ & $y_{2}(t, 1)$ & $y_{1}^{\prime \prime}(t, 1)$ & 7.99234 \\
\hline 0.015625 & -0.153774 & -0.054983 & 9.74817 & \\
0.140625 & -0.912267 & -0.822269 & 12.0285 & 9.86412 \\
0.265625 & -1.51779 & -1.43476 & 14.6623 & 12.1679 \\
0.390625 & -1.93488 & -1.85664 & 17.6034 & 14.8303 \\
0.515625 & -2.12245 & -2.04637 & 20.8309 & 21.0706 \\
0.640625 & -2.03457 & -1.95749 & 24.3424 & 24.6248 \\
0.765625 & -1.62082 & -1.53898 & 28.1502 & 28.4796 \\
0.890625 & -0.826309 & -0.7353 & 31.2141 \\
0.984375 & 0.0550747 & 0.1563 & & 31.5816 \\
\hline
\end{tabular}

$0.2(1-t)$ and we can find out the solution approximately by using scheme of successive approximation.

We have presented the numerical result of Example 2 by using proposed method in Tables 1 and 2 . Table 1 is the numerical results for Example 2 when the level value is equal to 1 and Table 2 is the numerical results when the level value is equal to 0.5 . The first rows of Tables 1 and 2 are the numeral values of the dependent variables and the second rows are the values of the lower functions and the third rows are the values of upper functions and the fourth and fifth rows are the values of lower and upper functions for $(1,1)$ derivatives of $y(t)$ for Example 2. For Tables 1 and 2, we can know that $y_{1}(t, r) \leq y_{2}(t, r)$ and $y_{1}^{\prime \prime}(t, r) \leq y_{2}^{\prime \prime}(t, r)$ have been satisfied. Also we can see that $y_{1}(t, r) \leq y_{2}(t, r)$ and $y_{1}^{\prime \prime}(t, r) \leq y_{2}^{\prime \prime}(t, r)$ have been satisfied for all $r \in$ $(0,1]$ and $y_{1}(t, r), y_{2}(t, r)$ constitute intervals. We compute by Mathematica6.0.

\section{Conclusion}

We have studied one condition for a two-point boundary value problem to have a solution and investigated one method to calculate the solution. In conclusion, we have to consider the cut-equations and problem (43) with the inequality condition to get the fuzzy solution. The conditions were given by the coefficients, boundary conditions, and nonhomogeneous terms of fuzzy differential equations. The next step in the research is to extend the results of this paper to generalized cases.

\section{Data Availability}

No data were used to support this study.

\section{Conflicts of Interest}

The authors declare that there are no conflicts of interest regarding the publication of this paper.

\section{Acknowledgments}

The authors would like to thank Professor Manoj Gauthaman, Editorial Office, for his consideration about our paper.

\section{References}

[1] H. Zarei, A. V. Kamyad, and A. A. Heydari, "Fuzzy modeling and control of HIV infection," Computational and Mathematical Methods in Medicine, vol. 2012, Article ID 893474, 17 pages, 2012.

[2] R. M. Jafelice, L. C. de Barros, R. C. Bassanezi, and F. Gomide, "Fuzzy modeling in symptomatic HIV virus infected population," Bulletin of Mathematical Biology, vol. 66, no. 6, pp. 15971620,2004

[3] S. Salahshour, A. Ahmadian, F. Ismail, and D. Baleanu, "A fractional derivative with non-singular kernel for interval-valued functions under uncertainty," Optik - International Journal for Light and Electron Optics, vol. 130, pp. 273-286, 2017.

[4] V. Lupulescu, "Fractional calculus for interval-valued functions," Fuzzy Sets and Systems, vol. 265, pp. 63-85, 2014. 
[5] S. Salahshour, T. Allahviranloo, and S. Abbasbandy, "Existence and uniqueness results for fractional differential equations with uncertainty," Advances in Difference Equations, vol. 2012, article $112,2012$.

[6] M. T. Malinowski, "Random fuzzy fractional integral equations-theoretical foundations," Fuzzy Sets and Systems, vol. 265, pp. 39-62, 2015.

[7] R. M. Jena and S. Chakraverty, "A new iterative method based solution for fractional Black-Scholes option pricing equations (BSOPE)," SN Applied Sciences, vol. 1, no. 1, p. 95, 2019.

[8] K. Sin, M. Chen, H. Choi, and K. Ri, "Fractional Jacobi operational matrix for solving fuzzy fractional differential equation 1," Journal of Intelligent \& Fuzzy Systems: Applications in Engineering and Technology, vol. 33, no. 2, pp. 1041-1052, 2017.

[9] K. Sin, M. Chen, C. Wu, K. R, and H. Choi, "Application of a spectral method to fractional differential equations under uncertainty," Journal of Intelligent \& Fuzzy Systems: Applications in Engineering and Technology, vol. 35, pp. 4821-4835, 2018.

[10] A. Ahmadian, F. Ismail, S. Salahshour, D. Baleanu, and F. Ghaemi, "Uncertain viscoelastic models with fractional order: a new spectral tau method to study the numerical simulations of the solution," Communications in Nonlinear Science and Numerical Simulation, vol. 53, pp. 44-64, 2017.

[11] A. Ahmadian, S. Salahshour, M. Ali-Akbari, F. Ismail, and D. Baleanu, "A novel approach to approximate fractional derivative with uncertain conditions," Chaos, Solitons \& Fractals, vol. 104, pp. 68-76, 2017.

[12] R. M. Jena, S. Chakraverty, and S. K. Jena, "Dynamic response analysis of fractionally damped beams subjected to external loads using homotopy analysis method," Journal of Applied and Computational Mechanics, vol. 5, no. 2, pp. 355-366, 2019.

[13] R. M. Jena and S. Chakraverty, "Solving time-fractional Navier-Stokes equations using homotopy perturbation Elzaki transform," SN Applied Sciences, vol. 1, no. 1, p. 16, 2019.

[14] S. Chakraverty, S. Tapaswini, and D. Behera, Fuzzy Arbitrary Order System:Fuzzy Fractional Differential Equations and Applications, John Wiley \& Sons, Inc., Hoboken, NJ, USA, 2016.

[15] S. Salahshour, A. Ahmadian, and D. Baleanu, "Variation of constant formula for the solution of interval differential equations of non-integer order," The European Physical Journal Special Topics, vol. 226, no. 16-18, pp. 3501-3512, 2017.

[16] D. O’Regan, V. Lakshmikantham, and J. J. Nieto, "Initial and boundary value problems for fuzzy differential equations," Nonlinear Analysis. Theory, Methods \& Applications, vol. 54, no. 3, pp. 405-415, 2003.

[17] V. Lakshmikantham, K. N. Murty, and J. Turner, "Two-point boundary value problems associated with non-linear fuzzy differential equations," Mathematical Inequalities \& Applications, vol. 4, no. 4, pp. 527-533, 2001.

[18] R. P. Agarwal, V. Lakshmikantham, and J. J. Nieto, "On the concept of solution for fractional differential equations with uncertainty," Nonlinear Analysis: Theory, Methods \& Applications, vol. 72, no. 6, pp. 2859-2862, 2010.

[19] P. Prakash, J. J. Nieto, S. Senthilvelavan, and G. Sudha Priya, "Fuzzy fractional initial value problem," Journal of Intelligent \& Fuzzy Systems: Applications in Engineering and Technology, vol. 28, no. 6, pp. 2691-2704, 2015.

[20] M. Mazandarani and A. V. Kamyad, "Modified fractional Euler method for solving fuzzy fractional initial value problem," Communications in Nonlinear Science and Numerical Simulation, vol. 18, no. 1, pp. 12-21, 2013.
[21] A. Khastan and J. J. Nieto, "A boundary value problem for second order fuzzy differential equations," Nonlinear Analysis: Theory, Methods \& Applications, vol. 72, no. 9-10, pp. 3583-3593, 2010.

[22] J. J. Nieto, R. Rodríguez-López, and M. Villanueva-Pesqueira, "Exact solution to the periodic boundary value problem for a first-order linear fuzzy differential equation with impulses," Fuzzy Optimization and Decision Making, vol. 10, no. 4, pp. 323339, 2011.

[23] H. V. Ngo, V. Ho, and M. D. Tran, "Fuzzy fractional differential equations under Caputo-Katugampola fractional derivative approach," Fuzzy Sets and Systems, 2018.

[24] H. V. Ngo, V. Lupulescu, and D. O'Regan, "A note on initial value problems for fractional fuzzy differential equations," Fuzzy Sets and Systems, vol. 347, pp. 54-69, 2018.

[25] Y. Wang, S. Sun, and Z. Han, "Existence of solutions to boundary value problems for a class of nonlinear fuzzy fractional differential equations," Advances in Analysis, vol. 2, no. 4, 2017.

[26] N. A. Gasilov, Ş. E. Amrahov, A. G. Fatullayev, and I. F. Hashimoglu, "Solution method for a boundary value problem with fuzzy forcing function," Information Sciences, vol. 317, pp. 349-368, 2015.

[27] B. Bede and L. Stefanini, "Generalized differentiability of fuzzyvalued functions," Fuzzy Sets and Systems, vol. 230, pp. 119-141, 2013.

[28] B. Bede and S. G. Gal, "Generalizations of the differentiability of fuzzy-number-valued functions with applications to fuzzy differential equations," Fuzzy Sets and Systems, vol. 151, no. 3, pp. 581-599, 2005.

[29] A. Khastan, F. Bahrami, and K. Ivaz, "New results on multiple solutions for $N$ th-order fuzzy differential equations under generalized differentiability," Boundary Value Problems, vol. 2009, Article ID 395714, 2009.

[30] S. Tapaswini, S. Chakraverty, and T. Allahviranloo, "A new approach to $n$th order fuzzy differential equations," Computational Mathematics and Modeling, vol. 28, no. 2, pp. 278-300, 2017.

[31] V. Lupulescu, L. S. Dong, and N. Van Hoa, "Existence and uniqueness of solutions for random fuzzy fractional integral and differential equations," Journal of Intelligent \& Fuzzy Systems: Applications in Engineering and Technology, vol. 29, no. 1, pp. 27-42, 2015. 


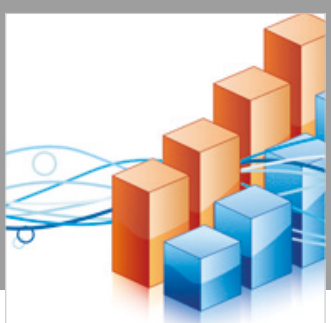

Advances in

Operations Research

\section{-n-m}
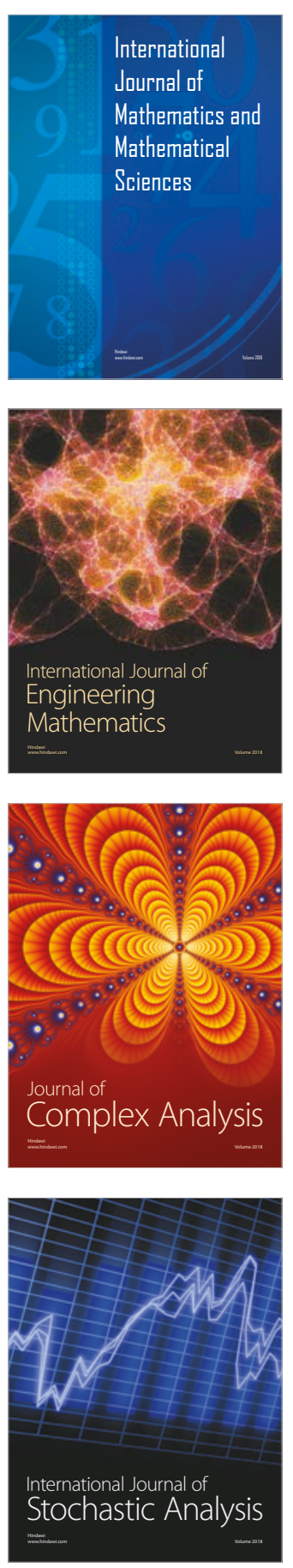
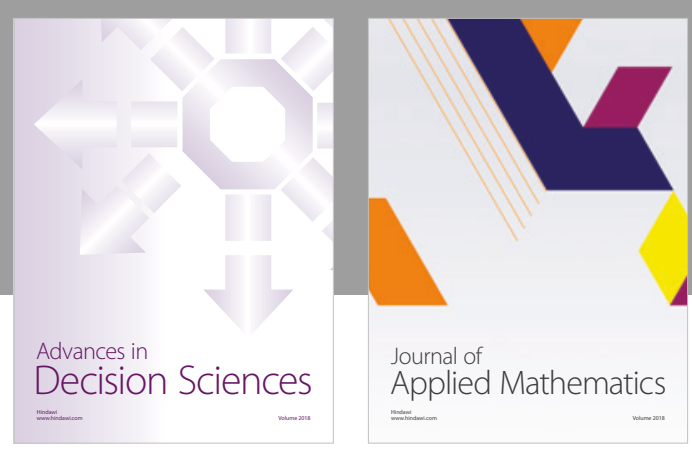

Journal of

Applied Mathematics
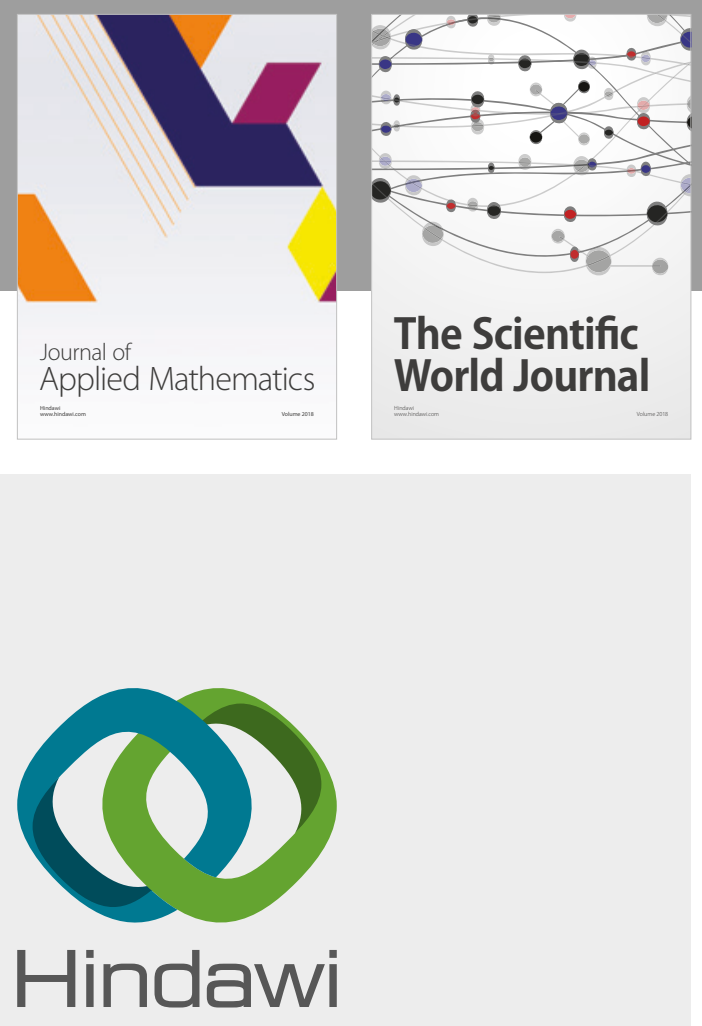

Submit your manuscripts at

www.hindawi.com

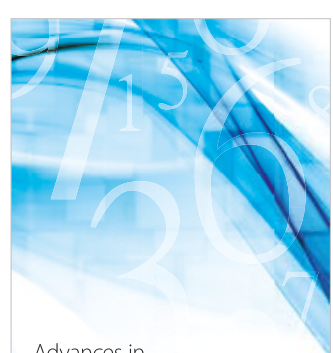

Advances in
Numerical Analysis
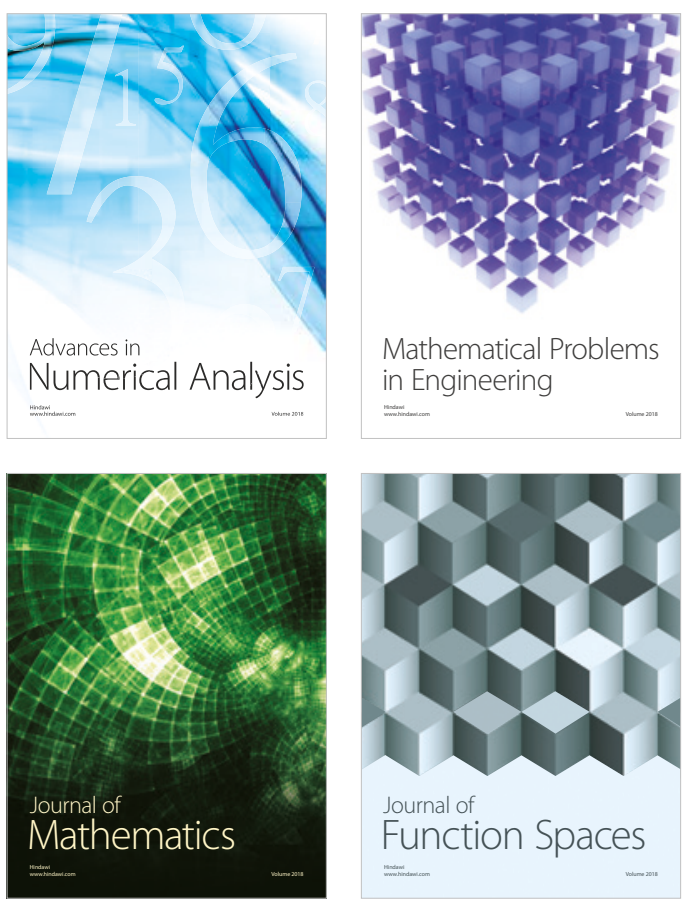

Mathematical Problems in Engineering

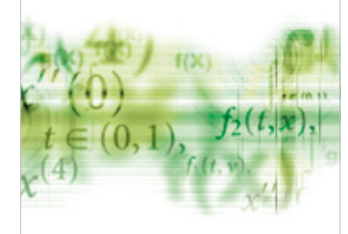

International Journal of

Differential Equations

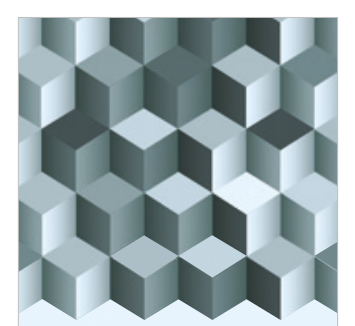

Journal of

Function Spaces

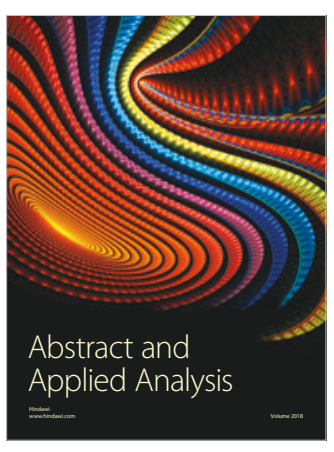

The Scientific

World Journal

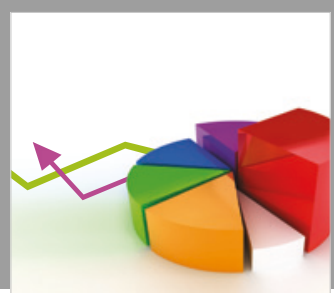

Journal of

Probability and Statistics
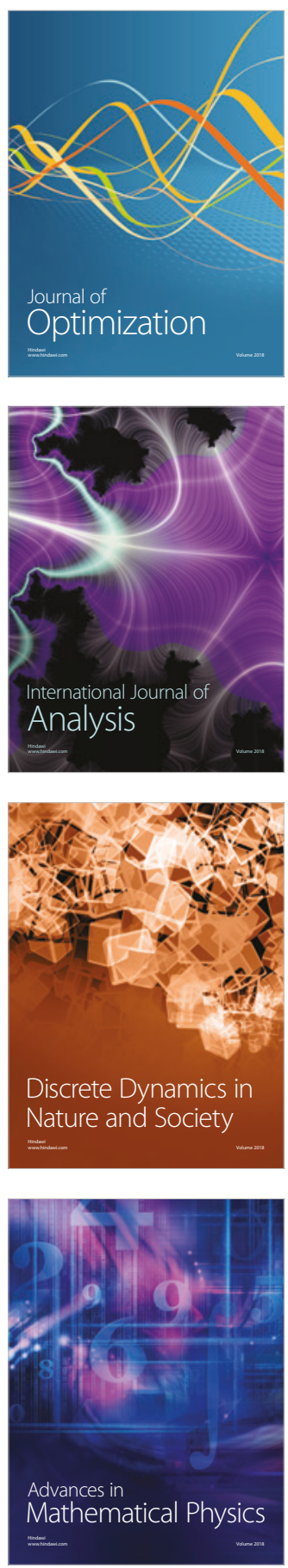